\title{
General Laws of Adaptation to Environmental Factors: from Ecological Stress to Financial Crisis
}

\author{
A.N. Gorban ${ }^{1}{ }^{*}$, E.V. Smirnova ${ }^{2}$ and T.A. Tyukina ${ }^{1}$ \\ ${ }^{1}$ Department of Mathematics, University of Leicester, Leicester, LE1 7RH, UK \\ ${ }^{2}$ Siberian Federal University, Krasnoyarsk, 660041, Russia
}

\begin{abstract}
We study ensembles of similar systems under load of environmental factors. The phenomenon of adaptation has similar properties for systems of different nature. Typically, when the load increases above some threshold, then the adapting systems become more different (variance increases), but the correlation increases too. If the stress continues to increase then the second threshold appears: the correlation achieves maximal value, and start to decrease, but the variance continue to increase. In many applications this second threshold is a signal of approaching of fatal outcome.

This effect is supported by many experiments and observation of groups of humans, mice, trees, grassy plants, and on financial time series. A general approach to explanation of the effect through dynamics of adaptation is developed. H. Selye introduced "adaptation energy" for explanation of adaptation phenomena. We formalize this approach in factors - resource models and develop hierarchy of models of adaptation. Different organization of interaction between factors (Liebig's versus synergistic systems) lead to different adaptation dynamics. This gives an explanation to qualitatively different dynamics of correlation under different types of load and to some deviation from the typical reaction to stress.

In addition to the "quasistatic" optimization factor - resource models, dynamical models of adaptation are developed, and a simple model (three variables) for adaptation to one factor load is formulated explicitly.
\end{abstract}

Key words: adaptation, factor, correlations, principal components, dynamics, crisis AMS subject classification: 92D40, 93C40, 91B84, 62M10

\footnotetext{
*Corresponding author. E-mail: ag153@le.ac.uk
} 


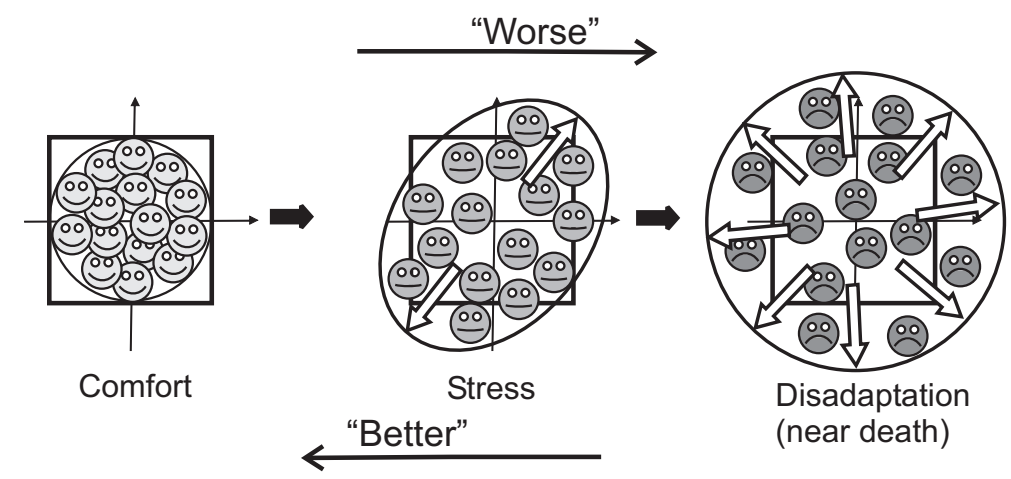

Figure 1: Correlations and variance in crisis. The typical picture: $\operatorname{Cor} \uparrow ; \operatorname{Var} \uparrow-$ stress; $\operatorname{Cor} \downarrow$ ; Var $\downarrow$ - recovering; Cor $\downarrow ; \operatorname{Var} \uparrow$ - approaching the disadaptation catastrophe after the bottom of the crisis.

\section{Introduction}

In many areas of practice, from physiology to economics, psychology, and engineering we have to analyze behavior of groups of many similar systems, which are adapting to the same or similar environment. Groups of humans in hard living conditions (Far North city, polar expedition, or a hospital, for example), trees under influence of anthropogenic air pollution, rats under poisoning, banks in financial crisis, enterprizes in recession, and many other situations of that type provide us with plenty of important problems, problems of diagnostics and prediction.

For many such situations it was found that the correlations between individual systems are better indicators than the value of attributes. More specifically, in thousands of experiments it was shown that in crisis, typically, even before obvious symptoms of crisis appear, the correlations increase, and, at the same time, variance (volatility) increases too. After the crisis achieves its bottom, it can develop into two directions: recovering (both correlations and variance decrease) or fatal catastrophe (correlations decrease, but variance continue to increase) (Fig. 1).

Now, after 21 years of studying of this effect $[19,50]$, we maintain that it is universal for groups of similar systems that are sustaining a stress and have an adaptation ability. Hence, a theory of an adequate level of universality is needed. On the other hand, situations with inverse behavior were predicted theoretically and found experimentally [28]. This makes the problem more intriguing.

In this paper we attempt to answer to this challenge. First we review and analyze data for different kinds of systems: humans [50, 43, 58, 59], mice [44], grassy plants [24], trees [55]. We perform also a case study of the thirty largest companies from the British stock market for the period 2006-2008.

In economics, we use also published results of data analysis for equity markets of seven major countries over the period 1960-1990 [27] and for the twelve largest European equity markets after the 1987 international equity market crash [35]. Some of results obtained in econophysics ([32]) also support our hypothesis [17, 42, 11, 30, 38, 45, 8].

There exists an important question about "crisis contagion" [33]: Do strong correlations appear before crisis and provide crisis contagion, or do they grow stronger because of crisis? Current 
trends in the network of stocks in the S\&P 500 and NASDAQ-100 indices during the credit crisis (2007-2008) demonstrate that the losses in certain markets, follow a cascade or epidemic flow along the correlations of various stocks. But whether or not this idea of epidemic or cascade is a metaphor or a causal model for this crisis is not so obvious [56].

Most of the data, we collected by ourselves or found in publications, support the hypothesis presented in Fig. 1. In all situations, the definitions of stress and crisis were constructed by experts in specific disciplines on the basis of specific knowledge. What do "better" and "worse" mean? This is a nontrivial special question and from the point of view of very practically oriented researchers the main outcome of modeling may be in the definition of crisis rather than in the explanation of details [12]. In many situations we can detect that one man's crisis is another man's road to prosperity.

Nevertheless, all the experiments are unbiased in the following sense: the definitions of the "better-worse" scale were done before the correlation analysis and did not depend on the results of that analysis. Hence, one can state, that the expert evaluation of the stress and crisis can be (typically) reproduced by the formal analysis of correlations and variances. Moreover, this analysis may give the symptoms of the future changes, before an obvious deterioration of the situation occur.

The basic model of such generality should include little detail, and we try to make it as simple as possible. We represent the systems, which are adapting to stress, as the systems which optimize distribution of available resource for neutralization of different aggressive factors (we consider deficit of anything needful as a negative factor too). These factor-resource models with optimization are very convenient for modeling of adaptation. We use a class of models many factors - one resource, but many nearest generalizations are absolutely clear (several resources, different models for the resource recovery, separation of the resource on a tactic resource and a strategic reserve, etc.). The crucial question is: what is the resource of adaptation? This question arose for the first time when Selye published the concept of adaptation energy and experimental evidence supporting this idea [51, 52]. After that, this notion was significantly improved [16], plenty of indirect evidence supporting this concept were found, but this elusive adaptation energy is still a theoretical concept, and in the modern "Encyclopedia of Stress" we read: "As for adaptation energy, Selye was never able to measure it..." [34]. Nevertheless, the notion of adaptation energy is very useful in analysis of adaptation and is now in wide use (see, for example, [1, 49]).

The question about the nature of adaptation resource remains important for the economic situation too. The idea of exchange helps here: any resource could be exchanged for another one, and the only question is - what is the "exchange rate", how fast this exchange could be done, what is the margin, how the margin depends on the exchange time, and what is the limit of that exchange. Market economics is much closer to the idea of resource universalization than biophysics and medicine are. It is possible to include the exchange processes into models, but many questions appear immediately about unknown coefficients. In the zero approximation we can just postulate the universal adaptation resource and hide all the exchange and recovering processes. For biophysics, this exchange idea seems also attractive, but of course there exist some limits of possible exchange of different resources. Nevertheless, we can follow the Selye arguments and postulate the adaptation energy under assumption that this is not an "energy" or Aristotle's "entelechy" (which 
definitely had an impact on Selye's work), but just a pool of various exchangeable resources. When an organism achieves the limits of resources exchangeability, the universal non-specific stress and adaptation syndrome transforms (disintegrates) into specific diseases. Near this limit we have to expect the critical retardation of exchange processes.

The idea of optimization is very natural for man-made systems in economics, but it requires usually some additional explanation in biophysics. The main source of the optimality idea in biology is the formalization of natural selection and adaptive dynamics. After works of Haldane (1932) [22] and Gause (1934) [14] this direction, with various concepts of fitness optimization, was further developed (see, for example, review papers [2, 37, 18]). To transfer the evolutionary optimality principles to the short and long term adaptation we need the idea of genocopy-phenocopy interchangeability ([60], p. 117). The phenotype modifications simulate the optimal genotype, but in a narrower interval of change. We can expect that adaptation also gives the optimal phenotype, but the interval of the possible changes should be even narrower, than for modifications. The idea of convergence of genetic and environmental effects was supported by analysis of genome regulation [61] (the principle of concentration-affinity equivalence). This gives a basis for the optimality assumption in adaptation modeling.

To analyze adaptation by optimality of resource redistribution we have to answer one more question: how is the system of factors organized? For arbitrary interactions between factors and organism the modeling will give an answer with a high level of uncertainty. To construct a specific function, we need much more data than it is possible to have in such a general situation. Ecology already has a very attractive version for an answer. This is Liebigs Law of the Minimum. The principle behind this law is quite simple. Originally, it meant that the scarcest necessity an organism requires will be the limiting factor to its performance. A bit more generally, the worst factor determines the situation for an organism, and free resource should, perhaps, be assigned for neutralization of that factor (until it loses its leadership). The opposite principle of factor organization is synergy: the superlinear mutual amplification of factors. Adaptation to Liebig's system of factors, or to any synergistic system, leads to two paradoxes of adaptation:

- Law of the Minimum paradox (Sec. 5.2.): If for a randomly selected pair, (State of environment - State of organism), the Law of the Minimum is valid (everything is limited by the factor with the worst value) then, after adaptation, many factors (the maximally possible amount of them) are equally important.

- Law of the Minimum inverse paradox (Sec. 5.3.): If for a randomly selected pair, (State of environment - State of organism), many factors are equally important and superlinearly amplify each other then, after adaptation, the smaller amount of factors is important (everything is limited by the factors with the worst non-compensated values, the system approaches the Law of the Minimum).

Perhaps the most realistic model is a system of factors, which consists of several synergistic groups, while interaction between groups satisfies Liebig's Law.

The optimization models of adaptation give the optimal distribution of the adaptation resource if a system has some amount of resource available and experiences given loads of environmental factors. But the situation changes in time continuously, and a dynamical theory of resource 
distribution is necessary. As a starting point for construction of dynamical models we select the biological ideas of H. Selye "adaptation energy" developed by B. Goldstone [16] from the "general practitioner" point of view. To formalize these ideas we used optimization approach together with formalism that we have adopted from the thermodynamic theory of affinity $[10,13]$.

A part of material was published in Ref. [21]. In this paper, we significantly extend analysis of destroying of correlations "on the other side of crisis", add new data about financial time series and a sketch of the theory of dynamical models of adaptation.

After introduction of the main ideas and data sources, we are in position to start more formal consideration.

\section{Indicators}

How can we measure correlations between various attributes in a population? If we have two variables, $x$ and $y$, the answer is simple: we measure $\left(x_{i}, y_{i}\right)$ for different individuals $(i=1, \ldots n$, $n>1$ is the number of measurements). The sample correlation coefficient (the Pearson coefficient) is

$$
r=\frac{\langle x y\rangle-\langle x\rangle\langle y\rangle}{\sqrt{\left\langle\left(x_{i}-\langle x\rangle\right)^{2}\right\rangle} \sqrt{\left\langle\left(y_{i}-\langle y\rangle\right)^{2}\right\rangle}}
$$

where $\langle\ldots\rangle$ stands for the sample average value: $\langle x\rangle=\frac{1}{n} \sum_{i} x_{i}$.

If individuals are characterized by more than two attributes $\left\{x^{l} \mid l=1, \ldots m\right\}$ then we have already $m(m-1) / 2$ correlation coefficients between them, $r_{j k}$. In biophysics, we usually analyze correlations between attributes, and each individual organism is represented as a vector of attributes values.

In econophysics, the standard situation is a "transposed" one: we study correlations between objects, and data vectors correspond to the moments in time (with a possible extension to the variables taken with some delay). Each object (stock, enterprize, ...) is represented as a vector of values of a variable (asset return, for example) in a window of time. This is, essentially, just a difference between $X$ and $X^{T}$, where $X$ is the matrix of data.

In correlation analysis, this difference appears in two operations: centralization (when we subtract means in computation of covariance) and normalization (when we transform the covariance into the correlation coefficient). In one case, we centralize and normalize the columns of $X$ : subtract average values in columns, and divide columns on their standard deviations. In another case, we apply these operations to the rows of $X$. For financial time series, the synchronous averages and variances ("varieties") and time averages and variances ("volatilities") have different statistical properties. This was clearly demonstrated in a special case study [25].

Nevertheless, such a difference does not appear very important for the analysis of the total level of correlations in crisis (just the magnitude of correlation changes, and correlations in time are uniformly less than synchronous ones, that is with agreement of observations from Ref. [25]). More details are presented in the special case study below. 
In our case study we demonstrated that in the analysis of financial time series it may be also convenient to study correlations between parameters, not between individuals. It means that we can study correlation between any two time moments and consider data from different individuals as values of random $2 \mathrm{D}$ vector. It is necessary to stress that this correlations between two time moments are very different from the standard autocorrelations for stationary time series (which characterize the sample of all pairs of time moments with a given lag in time).

For example, let $X_{i t}$ be a log-return value for $i$ th stock at time moment $t(i=1, \ldots n, t=$ $\tau+1, \ldots \tau+T)$. Each row of the data matrix $X_{i t}$ corresponds to an individual stock and each column corresponds to a time moment. If we normalize and centralize data in rows and calculate the correlation coefficients between rows $\left(r_{i j}=\sum_{t} X_{i t} X_{j t}\right.$ for centralized and normalized data) then we find the correlations between stocks. If we normalize and centralize data in columns and calculate the correlation coefficients between them $\left(r_{t_{1} t_{2}}=\sum_{i} X_{i t_{1}} X_{i t_{2}}\right.$ for centralized and normalized data) then we find the correlations between time moments. In crisis, dynamics of the correlations between stocks is similar to behavior of the correlations between time moments. One benefit from use of the correlations between time moment is absence of averaging in time (locality): this correlation coefficient depends on data at two time moments. This allows to analyze the anatomy of crisis in time.

The sample correlation coefficient (2.1) can be used as an estimate of a "proper" correlation between random variables. But one can use the matrix of the sample correlations, its eigenvectors and eigenvalues as characteristics of linear connections in a finite dataset, without any probabilistic hypothesis, in the spirit of the classical Pearson work [41] for example. Here we study the properties of finite datasets.

To collect information about correlations between many attributes in one indicator, it is possible to use various approaches. Fist of all, we can evaluate the non-diagonal part of the correlation matrix in any norm, for example, in $L_{p}$ norm

$$
\|r\|_{p}=\left(\sum_{j>k}\left(r_{j k}\right)^{p}\right)^{\frac{1}{p}} .
$$

If one would like to study strong correlations, then it may be better to delete terms with values below a threshold $\alpha>0$ from this sum:

$$
G_{p, \alpha}=\left(\sum_{j>k,\left|r_{j k}\right|>\alpha}\left(r_{j k}\right)^{p}\right)^{\frac{1}{p}} .
$$

This quantity $G_{p, \alpha}$ is a $p$-weight of the $\alpha$-correlation graph. The vertices of this graph correspond to variables, and these vertices are connected by edges, if the absolute value of the correspondent sample correlation coefficient exceeds $\alpha:\left|r_{j k}\right|>\alpha$. In practice, the most used indicator is the weight $G=G_{1,0.5}$, which corresponds to $p=1$ and $\alpha=0.5$.

Another group of indicators is produced from the principal components of the data. The principal components are eigenvectors of the covariance matrix and depend on the scales. Under normalization of scales to unit variance, we deal with the correlation matrix. Let $\lambda_{1} \geq \lambda_{2} \geq \ldots \lambda_{m} \geq 0$ 
be eigenvalues of the correlation matrix. In this paper, we use the eigenvalues and eigenvectors of the correlation matrix. It is obvious that $\langle\lambda\rangle=1$ and $m^{p-1} \geq\left\langle\lambda^{p}\right\rangle \geq 1$ for $p>1,\left\langle\lambda^{p}\right\rangle=1$ if all non-diagonal correlation coefficients are zero and $\left\langle\lambda^{p}\right\rangle=m^{p-1}$ if all correlation coefficients are \pm 1 . To select the dominant part of principal components, it is necessary to separate the "random" part from the "non-random" part of them. This separation was widely discussed (see, for example, the expository review [6]).

The simplest decision gives Kaiser's significance rule: the significant eigenvalues are those, which are greater than the average value: $\left.\lambda_{i}\right\rangle\langle\lambda\rangle$. For the eigenvalues of the correlation matrix which we study here, it means $\lambda_{i}>1$. This rule works reasonable, when there are several eigenvalues significantly greater than one and others are smaller, but for a matrix which is close to a random matrix the performance may not be so good. In such cases this method overestimates the number of principal components.

In econophysics, another simple criterion for selection of dominant eigenvalues became popu$\operatorname{lar}[53,42,45,8]$. Let us imagine that the dimension of the data vector $m$ is large and the amount of data points $n$ is also large, but their ratio $q=n / m$ is not. This is the typical situation when we analyze data about thousands of stocks: in this case the time window could not be much larger than the dimension of data vector. Let us compare our analysis of real correlations to the fictitious correlations, which appear in $m \times n$ data matrices with independent, centralized, normalized and Gaussian matrix elements. If $n \rightarrow \infty$ for given $m$ then those fictitious correlations disappear, but if both $m, n \rightarrow \infty$ for constant $q>1$ then there exists the limit distribution of eigenvalues $\lambda$ with density

$$
\begin{aligned}
& \rho(\lambda)=\frac{q}{2 \pi} \sqrt{\left(\frac{\lambda_{\max }}{\lambda}-1\right)\left(1-\frac{\lambda_{\min }}{\lambda}\right)} ; \lambda_{\min } \leq \lambda \leq \lambda_{\max } \\
& \lambda_{\max / \min }=1+\frac{1}{q} \pm 2 \sqrt{\frac{1}{q}} .
\end{aligned}
$$

If the amount of points is less than dimension of data, $(q<1)$ the same formula with substitution of $q$ by $1 / q$ is valid for distribution of non-zero eigenvalues.

Instead of Kaiser's rule for dominant eigenvalues of the correlation matrix we get $\lambda_{i}>\lambda_{\max }$ with $\lambda_{\max }$ given by Eq. (2.4). If $q$ grows to $\infty$, this new rule turns back into Kaiser's rule. If $q$ is minimal $(q=1)$, then the proposed change of Kaiser's rule is maximal, $\lambda_{\max }=4$ and for dominant eigenvalues of the correlation matrix it should be $\lambda_{i}>4$. This new estimate is just an analogue of Kaiser's rule in the case when the amount of data vectors is compatible with the dimension of the data space, and, therefore, the data set is far from the law of large numbers conditions.

Another popular criterion for selection of dominant eigenvalues gives the so-called broken stick model. Consider the closed interval $J=[0,1]$. Suppose $J$ is partitioned into $m$ subintervals by randomly selecting $m-1$ points from a uniform distribution in the same interval. Denote by $l_{k}$ the length of the $k$-th subinterval in the descending order. Then the expected value of $l_{k}$ is

$$
\mathbf{E}\left(l_{k}\right)=\frac{1}{m} \sum_{j=k}^{m} \frac{1}{j} .
$$


Following the broken stick model, we have to include into the dominant part those eigenvalues $\lambda_{k}$ (principal components), for which

$$
\frac{\lambda_{1}}{\sum_{i} \lambda_{i}}>\mathbf{E}\left(l_{1}\right) \& \frac{\lambda_{2}}{\sum_{i} \lambda_{i}}>\mathbf{E}\left(l_{2}\right) \& \ldots \& \frac{\lambda_{k}}{\sum_{i} \lambda_{i}}>\mathbf{E}\left(l_{k}\right)
$$

If the amount of datavectors $n$ is less than the data dimension $m$, then $m-n$ eigenvalues are zeros, and in Eqs. (2.5), (2.6) one should take $n$ subintervals instead of $m$ ones.

It is worth to mention that the trace of the correlation matrix is $m$, and the broken stick model transforms (for $m>n$ ) into $\lambda_{i}>\sum_{j=i}^{m} \frac{1}{j}(i=1, \ldots k)$. From the practical point of view, this method slightly underestimates the number of dominant eigenvalues. There are other methods based on the random matrices ensembles, but nobody knows the exact dimension of the empirical data, and the broken stick model works satisfactory and remains "the method of choice".

To compare the broken stick model to Kaiser's rule, let us mention that the first principal component is always significant due to Kaiser's rule (if there exists at least one nonzero correlation coefficient), but due to the broken stick model it should be sufficiently large: the inequality $\lambda_{1}>$ $\sum_{j=1}^{m} \frac{1}{j}$ should hold. In a high dimension $m$ we can approximate the sum by the quadrature: $\lambda_{1} \gtrsim \ln m$.

If we have the dominant eigenvalues, $\lambda_{1} \geq \lambda_{2} \geq \ldots \lambda_{l}>0, l<m$, then we can produce some other measures of the sample correlation:

$$
\frac{\lambda_{1}}{\lambda_{l}} ; \sum_{j=1}^{l-1} \frac{\lambda_{j}}{\lambda_{j+1}} ; \quad \frac{1}{m} \sum_{j=1}^{l} \lambda_{j} .
$$

Together with $\left\langle\lambda^{p}\right\rangle(p>1$, the usual choice is $p=2)$ this system of indicators can be used for analysis of empirical correlations.

The choice of possible indicators is very rich, but happily, many case studies have shown that in analysis of adaptation the simplest weight $G$ of the correlation graph performs well (better or not worse than all other indicators - see the case study below). The only adjustment which may slightly improve performance of the indicator, is optimization of selection of the threshold $\alpha$.

A similar observation was made in Ref. [38]. There the "asset tree" was studied, that is the recently introduced minimum spanning tree description of correlations between stocks. The mean of the normalized dynamic asset tree lengths was considered as a promising indicator of the market dynamics. It appeared that a simple average correlation coefficient gives the same signal in time, as a more sophisticated indicator, the mean of the normalized dynamic asset tree lengths. (compare Fig. 1 and Fig. 2 from Ref. [38]). Especially we would like to attract attention to Fig. 12 from that paper, where very similar behavior of the mean correlation coefficient, the normalized tree length, and the risk of the minimum risk portfolio, as functions of time, was demonstrated.

In many publications in econophysics the average correlation coefficient is used instead of sums of absolute values in Eq. (2.3). This is possible because in many financial applications the orientation of the scales is fixed and the difference between positive and negative correlations is very important, for example, for portfolio optimization. In a more general situation we have to use absolute values because we cannot coordinate a priori direction of different axes. 

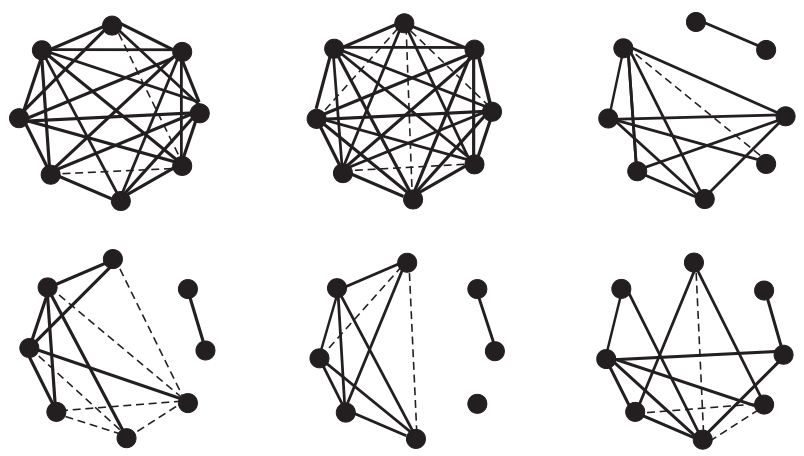

Figure 2: Correlation graphs of lipid metabolism for newborn babies. Vertices correspond to different fractions of lipids, solid lines correspond to correlation coefficient between fractions $\left|r_{i j}\right| \geq 0.5$, dashed lines correspond to $0.5>\left|r_{i j}\right| \geq 0.25$. Upper row - Far North, lower row - the temperate belt of Siberia (the comfort zone). From the left to the right: 1 st-3rd days, 4th-6th days, 7 th-10th days.

\section{Correlation and Risk in Physiology. Empirical Data}

In this section we represent some data which support our initial hypothesis (Fig. 1).

\subsection{Humans}

\subsubsection{Lipid Metabolism: Newborn children, Far North Versus Temperate Belt}

We studied lipid metabolism for healthy newborn babies born in the temperate belt of Siberia (the comfort zone) and in the migrant families of the same ethnic origin in a Far North city ${ }^{\dagger}$. The blood analysis was taken in the morning, on an empty stomach, at the same time each day. All the data were collected during the summer. Eight lipid fractions were analyzed [19]. Resulting correlation graphs are presented in Fig. 2. Here in correlation graphs solid lines represent correlation coefficient $\left|r_{i j}\right| \geq 0.5$, dashed lines represent correlation coefficient $0.5>\left|r_{i j}\right| \geq 0.25$.

\subsubsection{Adaptation of Adults for Change of Climatic Zone}

Activity of enzymes in human leukocytes was studied $[4,3]$. We analyzed the short-term adaptation (20 days) of groups of healthy 20-30 year old men who change their climate zone:

- From the temperate belt of Siberia (Krasnoyarsk, comfort zone) to Far North in summer and in winter;

\footnotetext{
${ }^{\dagger}$ The parents lived there in the standard city conditions.
} 


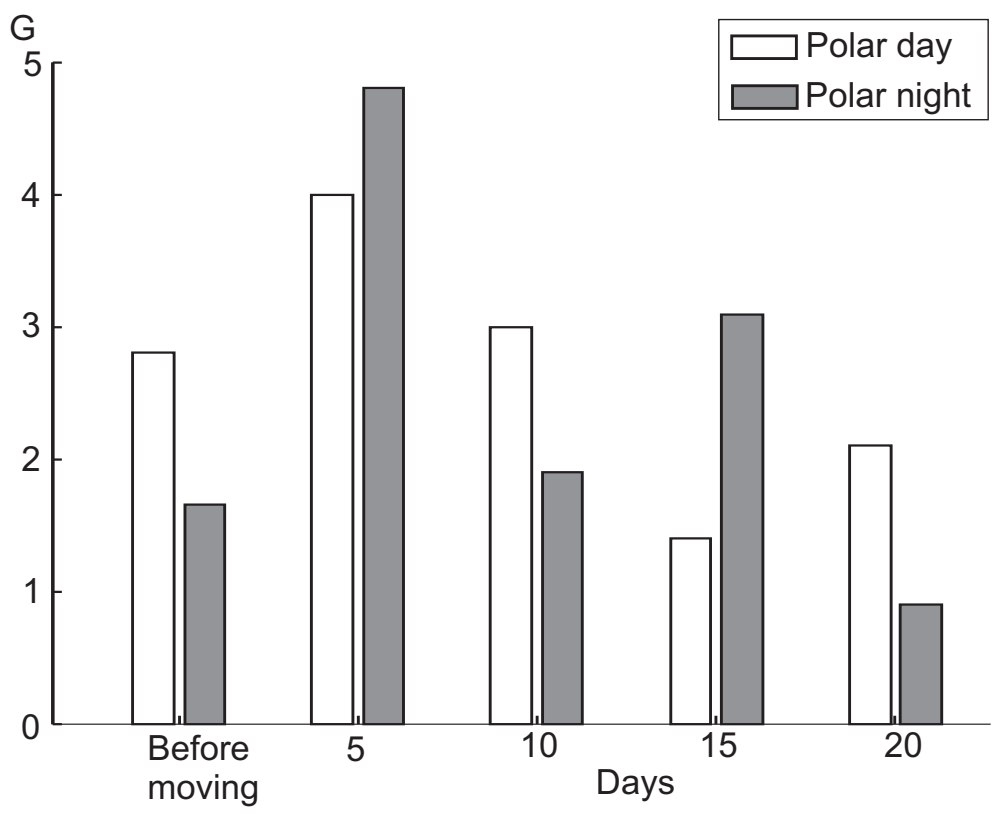

Figure 3: Weight of the correlation graphs of activity of enzymes in leucocytes during urgent adaptation to the Far North conditions. The non-monotonic character of adaptation is clear (there are the "waves of adaptation").

- From Far North to the South resort (Sochi, Black Sea) in summer;

- From the temperate belt of Russia to the South resort (Sochi, Black Sea) in summer.

Results are represented in Figs. 3,4. This analysis supports the basic hypothesis and, on the other hand, could be used for prediction of the most dangerous periods in adaptation, which need special care.

We selected the subgroup of people moved to Far North, that had any illness during the period of short-term adaptation. After 6 month at Far North, this group demonstrates much higher correlations between activity of enzymes in leucocytes than the main group: $G=5.81$ versus $G=1.36$ in the control group.

\subsubsection{Obesity and Treatment}

Obesity is a serious problem of contemporary medicine in developed countries. The study was conducted on patients (more than 70 people) with different heaviness of obesity [59]. The patients were divided into three groups.

First group included patients with first degree obesity and with second degree obesity, but without additional pathology. Second group consisted of patients with first and second group obesity and additional functional pathology (dyskinesia of digestive system, first degree hypertension, or asthenic syndrome). Third group included patients with first and second group obesity 


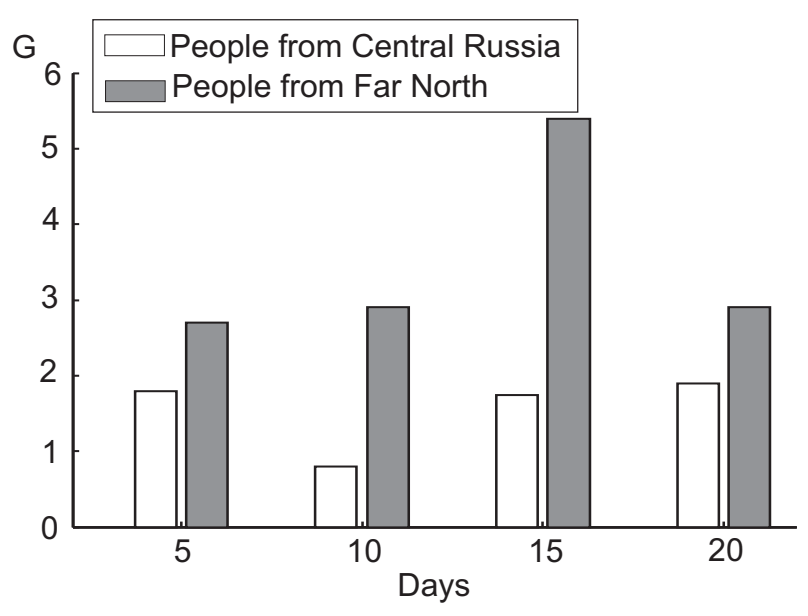

Figure 4: Weight of the correlation graphs of activity of enzymes in leucocytes during urgent adaptation at a resort. For people from Far North, the adaptation crisis occurs near the 15th day.

Table 1: Weight $G$ of the correlation graph for different groups of obese patients before and after 30 day complex treatment

\begin{tabular}{|c|c|c|}
\hline Group \# & $G$ before treatment & $G$ after treatment \\
\hline 1 & 7.29 & 6.63 \\
2 & 9.93 & 7.49 \\
3 & 12.99 & 10.03 \\
\hline
\end{tabular}

supplemented by organic pathology (peptic ulcer disease, second or third degree hypertension, myocardial infarction or brain stroke in anamnesis). Database with 50 attributes was studied (blood morphology, cholesterol level including fractions, creatinine, urea).

During 30 days patients received the standard treatment consisting of a diet, physical activity, pharmacological treatment, physical therapy and hydrotherapy. It was shown that the weight of the correlation graph $G$ of more informative parameters was originally high and dependent on the heaviness of sickness and was decreased during therapy (Table 1).

\subsubsection{Correlation Adaptometry at Work: Sport in Far North}

Presented data and dozens of other experiments ensured us that the effect is universal and it gives us a method to compare groups of people in various situations. This method received the name "Correlation adaptometry". An example of application of this method gives us the following problem. How sport interacts with ecological stress? More specifically, Dr. S.Y. Skobeleva and Prof. L.B. Zakharova (at the Institute for Medical Problems of Northern Regions, Krasnoyarsk) collected data about schoolchildren (primary school) from Far North and from the temperate belt of Siberia, who did sport intensively (swimming in swimming pools) and about a control group of schoolchildren, who did not work out intensively. Results are represented in Fig. 5. As a result it was concluded that the same intensity of the same sport could have the opposite effects on health 


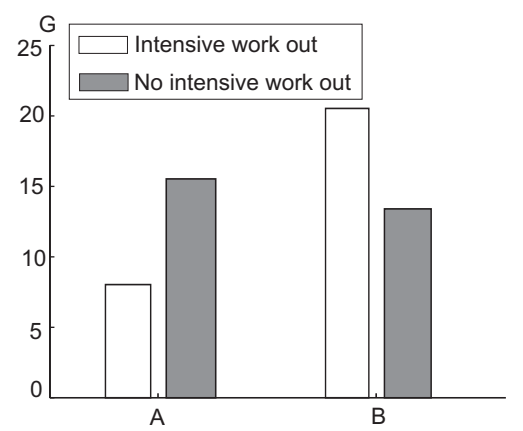

Figure 5: Weight of the correlation graphs of activity of enzymes in leucocytes for schoolchildren (primary school). A - temperate belt of Siberia, B - Far North.

(in perspective) in the temperate belt and in Far North.

\subsection{Destroying of Correlations "on the Other Side of Crisis"}

It is very important to understand where the system is going: (i) to the bottom of the crisis with possibility to recover after that bottom, (ii) to the normal state, from the bottom, or (iii) to the "no return" point, after which it cannot recover.

This problem was studied in many situation with analysis of fatal outcomes in oncological [29] and cardiological [57] clinics, and also in special experiments with acute hemolytic anemia caused by phenylhydrazine in mice [44]. The main result is presented in Fig. 1: when approaching the no-return point, correlations destroy ( $G$ decreases), and variance typically does continue to increase.

There exist no formal criterion to recognize the situation "on the other side of crisis". Nevertheless, it is necessary to select situations for testing of our hypothesis. Here can help the "general practitioner point of view" [16] based on practical experience. From such a point of view, three situations described below are on the other side of crisis: first 10 days after myocardial infarction, first 12 days of aftersurgial rehabilitations of patients with cancer of $3 \mathrm{~d}$ and 4 th clinical groups, and acute hemolytic anemia caused by phenylhydrazine in mice with lethal outcome.

\subsubsection{Fatal and Survival Outcomes after Myocardial Infarction}

Dynamics of correlations between physiological parameters after myocardial infarction (more than 50 cases in groups) was studied in Ref. [57]. For each patient, three groups of parameters were measured: echocardiography-derived variables (end-systolic and end-diastolic indexes, stroke index, and ejection fraction), parameters of central hemodynamics (systolic and diastolic arterial pressure, stroke volume, heart rate, the minute circulation volume, and specific peripheral resistance), biochemical parameters (lactate dehydrogenase, the heart isoenzyme of lactate dehydrogenase LDH1, aspartate transaminase, and alanine transaminase), and also leucocytes. Two groups were analyzed after 10 days of monitoring: the patients with lethal outcome, and the patients with survival outcome (with compatible amounts of group members). These groups do not differ signif- 


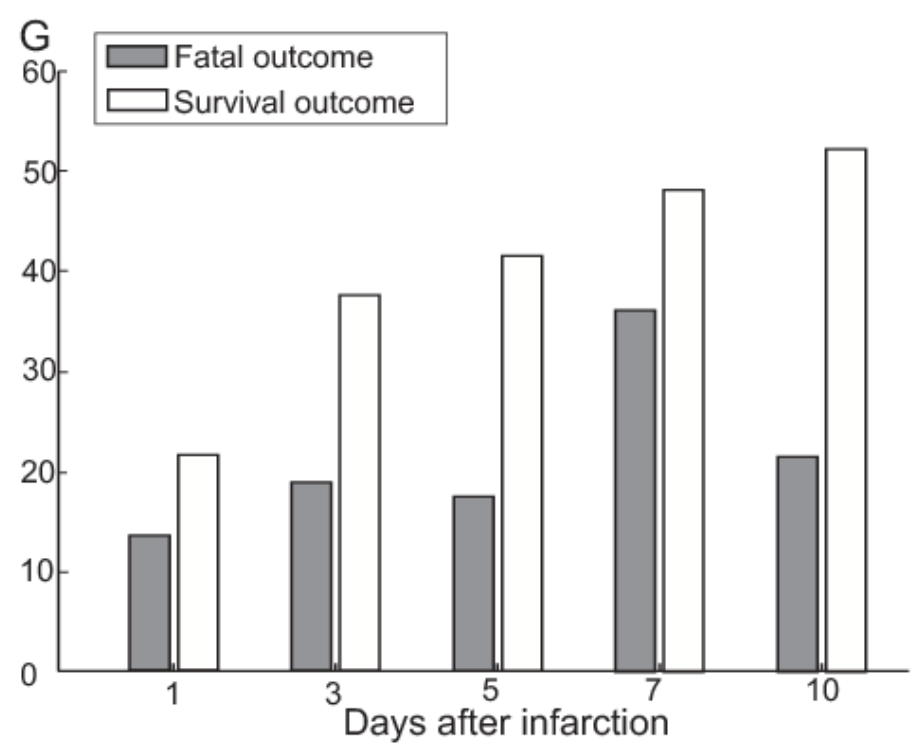

Figure 6: Dynamics of weight of the correlation graphs of echocardiography-derived variables (end-systolic and end-diastolic indexes, stroke index, and ejection fraction), parameters of central hemodynamics (systolic and diastolic arterial pressure, stroke volume, heart rate, the minute circulation volume, and specific peripheral resistance), biochemical parameters (lactate dehydrogenase, the heart isoenzyme of lactate dehydrogenase LDH1, aspartate transaminase, and alanine transaminase), and also leucocytes during 10 days after myocardial infarction for two groups of patients: for the survival outcome and for the fatal outcome. Here $G$ is the sum of the strongest correlations $\left|r_{i j}\right|>0.4, i \neq j[57]$.

icantly in average values of parameters and are not separable in the space of measured attributes. Nevertheless, dynamics of the correlations in the groups is essentially different. For the fatal outcome correlations were stably low (with a short pulse at the 7th day), for the survival outcome, the correlations were higher and monotonically grew (Fig 6).

Topologically, the correlation graph for the survival outcome included two persistent triangles with strong correlations: the central hemodynamics triangle, minute circulation volume - stroke volume - specific peripheral resistance, and the heart hemodynamics triangle, specific peripheral resistance - stroke index - end-diastolic indexes. The group with fatal outcome had not such persistent triangles in the correlation graph.

\subsubsection{Mortality and Correlations During the First 12 Days After Surgical Operation of Patients With Cancer}

The groups of people being operated on carcinoma of stomach, gullet cancer of III clinical group or lung carcinoma of III (100 people) and IV (60 people) clinical groups were examined during the period of rehabilitation after surgical treatment. The following indices were analyzed from 


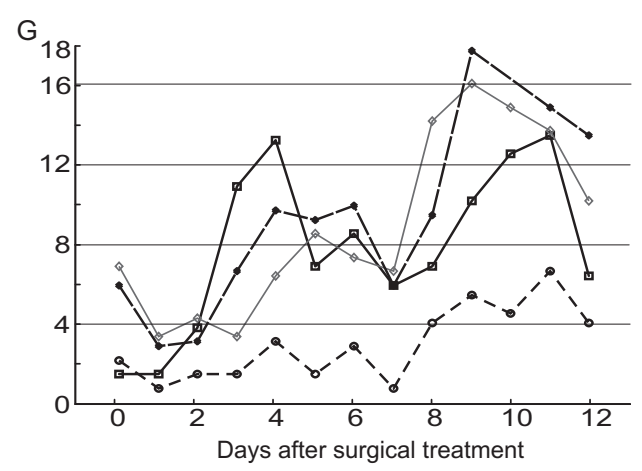

a)

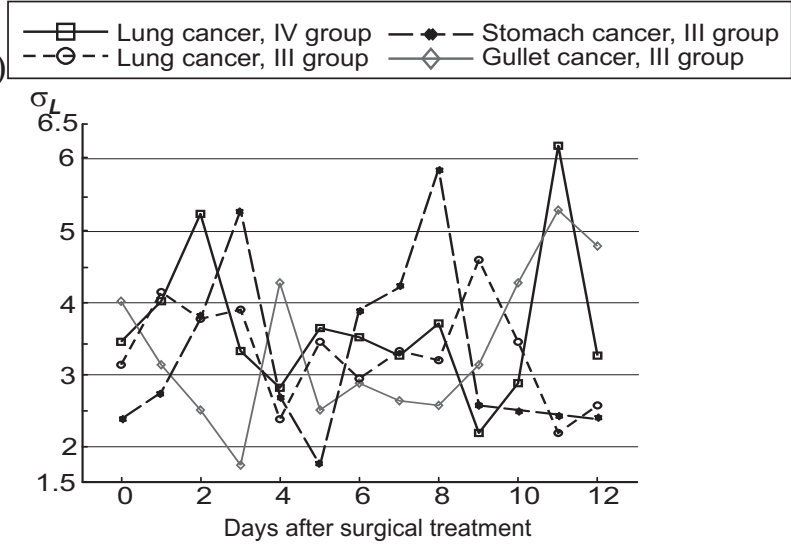

b)

$-\square-$ Lung cancer, IV group $-\leftarrow-$ Stomach cancer, III group
$--\Theta-$ - Lung cancer, III group $-\curvearrowright$ Gullet cancer, III group

Figure 7: Dynamics of weight of the correlation graph of the blood system variables (leukocytes, eosinophils, monocytes, lymphocytes, segmented neutrophils, band neutrophils and hemoglobin level) (a) and the standard deviation of leukocytes counts (b) during 12 days after surgical operation.

each group of people: cells counts (leukocytes, eosinophils, monocytes, lymphocytes, segmented neutrophils, and band neutrophils) and hemoglobin level.

It was noticed that the decease factors and surgical treatment are interact synergetically, in particular, they enhance each other influence on the organism.

Dynamics of the variance of blood cell counts and hemoglobin level goes during the first 10 days approximately in antiphase with dynamics of the weight of the correlation graph. For example, the weight of the correlation graph increases on the 4th and 6th days and decrease on the 5th and 7 th days after operation (Fig. 7a). On the other hand, the variance of blood cell counts and hemoglobin level achieves its maximal values on the 5th and 7th days and the minimal values on the 4th and 6th (Fig. 7b). The 5th and 7th days correspond to the maximum of mortality (there exists some difference between different types of cancer, both in mortality and correlations, Fig. 7). For oncologic patients, days of minimum weight of correlation graph can be critical points. On the 11th-12th days dynamics of the weight of the correlation graph and of the blood cell counts variance (for survived patients) start to go approximately in phase with the correlation graph (they 


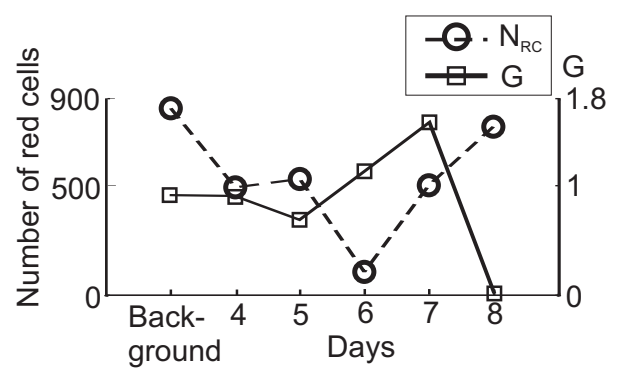

Figure 8: Adaptation and disadaptation dynamics for mice after phenylhydrazine injection.

decrease together). It can be interpreted as the passage through the bottom of crisis to the recovery side (Fig. 1).

\subsubsection{Acute Hemolytic Anemia in Mice}

This effect was demonstrated also in special experiments [44]. Acute hemolytic anemia caused by phenylhydrazine was studied in CBAxlac mice. After phenylhydrazine injections $(60 \mathrm{mg} / \mathrm{kg}$, twice a day, with interval 12 hours) during first 5-6 days the amount of red cells decreased (Fig. 8), but at the 7th and 8th days this amount increased because of spleen activity. After 8 days most of the mice died. Dynamics of correlation between hematocrit, reticulocytes, erythrocytes, and leukocytes in blood is presented in Fig. 8. Weight of the correlation graph increase precedeed the active adaptation response, but $G$ decreased to zero before death (Fig. 8), while amount of red cells increased also at the last day.

\subsection{Correlations and Health of Plants}

\subsubsection{Scots Pines Near a Coal Power Station}

The effect (Fig. 1) exists for plants too. The impact of emissions from a heat power station on Scots pine was studied [55]. For diagnostic purposes the secondary metabolites of phenolic nature were used. They are much more stable than the primary products and hold the information about past impact of environment on the plant organism for longer time.

The test group consisted of 10 Scots pines (Pinus sylvestric L) in a 40 year old stand of the II class in the emission tongue $10 \mathrm{~km}$ from the power station. The station had been operating on brown coal for 45 years. The control group of 10 Scots pines was from a stand of the same age and forest type, growing outside the industrial emission area. The needles for analysis were one year old from the shoots in the middle part of the crown. The samples were taken in spring in bud swelling period. Individual composition of the alcohol extract of needles was studied by high efficiency liquid chromatography. 26 individual phenolic compounds were identified for all samples and used in analysis.

No reliable difference was found in the test group and control group average compositions. For example, the results for Proantocyanidin content ( $\mathrm{mg} / \mathrm{g}$ dry weight) were as follows: 
Table 2: Weight $G$ of the correlation graph for different grassy plants under various trampling load

\begin{tabular}{|l|c|c|c|}
\hline Grassy Plant & Group 1 & Group 2 & Group 3 \\
\hline Lamiastrum & 1.4 & 5.2 & 6.2 \\
Paris (quadrifolia) & 4.1 & 7.6 & 14.8 \\
Convallaria & 5.4 & 7.9 & 10.1 \\
Anemone & 8.1 & 12.5 & 15.8 \\
Pulmonaria & 8.8 & 11.9 & 15.1 \\
Asarum & 10.3 & 15.4 & 19.5 \\
\hline
\end{tabular}

- Total $37.4 \pm 3.2$ (test) versus $36.8 \pm 2.0$ (control);

- Free $8.1 \pm 0.7$ (test) versus $8.0 \pm 2.0$ (control);

- Bound $29.3 \pm 3.2$ (test) versus $28.7 \pm 1.9$ (control).

Nevertheless, the variance of compositions of individual compounds in the test group was significantly higher, and the difference in correlations was huge: $G=17.29$ for the test group versus $G=3.79$ in the control group.

\subsubsection{Grassy Plants Under Trampling Load}

The grassy plants in oak tree-plants are studied. For analysis the fragments of forests are selected, where the densities of trees and bushes were the same. The difference between those fragments was in damaging of the soil surface by trampling. Tree groups of fragments are studied:

- Group 1 - no fully destroyed soil surface;

- Group $2-25 \%$ of soil surface are destroyed by trampling;

- Group 3-70\% of soil surface are destroyed by trampling.

The studied physiological attributes were: the height of sprouts, the length of roots, the diameter of roots, the amount of roots, the area of leafs, the area of roots. Results are presented in Table 2.

\section{Correlations and Risk in Economics. Empirical Data}

\subsection{Thirty Companies from the FTSE 100 Index. A Case Study}

\subsubsection{Data and Indicators}

For the analysis of correlations in financial systems we used the daily closing values over the time period 03.01.2006 - 20.11.2008 for companies that are registered in the FTSE 100 index 
(Financial Times Stock Exchange Index). The FTSE 100 is a market-capitalization weighted index representing the performance of the 100 largest UK-domiciled blue chip companies which pass screening for size and liquidity. The index represents approximately $88.03 \%$ of the UKs market capitalization. FTSE 100 constituents are all traded on the London Stock Exchanges SETS trading system. We selected 30 companies that had the highest value of the capital (on the 1st of January 2007) and stand for different types of business as well. The list of the companies and business types is displayed in Table 3.

Data for these companies are available form the Yahoo!Finance web-site. For data cleaning we use also information for the selected period available at the London Stock Exchange web-site. Let $x_{i}(t)$ denote the closing stock price for the $i$ th company at the moment $t$, where $i=\overline{1,30}$, $t=\overline{1,732}$. We analyze the correlations of logarithmic returns: $x_{i}^{l}(t)=\ln \frac{x_{i}(t)}{x_{i}(t-1)}, t=\overline{2,732}$ in sliding time windows of length $p=20$, this corresponds approximately to 4 weeks of 5 trading days, $t=\overline{p+1,732}$. The correlation coefficients $r_{i j}(t)$ and all indicators for time moment $t$ are calculated in the time window $[t-p, t-1]$, which precedees $t$. This is important if we would like to consider changes in these indicators as precursors of crisis.

The information about the level of correlations could be represented in several ways. Here we compare 4 indicators:

- The non-diagonal part of the correlation matrix in $L_{2}$ norm - $\|r\|_{2}$;

- The non-diagonal part of the correlation matrix in $L_{1}$ norm - $\|r\|_{1}$;

- The sum of the strongest elements $G=\sum_{j>i,\left|r_{i j}\right|>0.5}\left|r_{i j}\right|$;

- The amount Dim (or $\operatorname{Dim}_{\mathrm{BS}}$ ) of principal components estimated due to the broken stick model;

- The amount Dim MP $_{\text {of }}$ principal components estimated due to the random matrix theory (2.4);

- The amount Dim $\mathrm{K}$ of principal components estimated by Kaiser's rule $\lambda_{i}>1$.

Dynamics of the first three indicators are quite similar. Scatter diagrams (Figs. 9, 10) demonstrate strong connection between the indicators. We used the weight of the correlation graph $G$ (the sum of the strongest correlations $\left|r_{i j}\right|>0.5, i \neq j$ ) for our further analysis.

Fig. 11 allows us to compare dynamics of correlation, dimension and variance to the value of FTSE100. The correlations increase when the market goes down and decrease when it recovers. Dynamics of the variance of log-returns has the same tendency. To analyze the critical periods in more detail, let us select several time intervals and several positions of the sliding window inside these intervals.

\subsubsection{Correlation Graphs for Companies}

We extracted intervals for more detailed analysis. The first interval, 10/04/2006 - 21/07/2006, represents the FTSE index decrease and restoration in spring and summer 2006. The second interval, 04/07/2007 - 25/10/2007, includes July-October 2007. The third interval, 02/06/2008 - 
Table 3: Thirty largest companies for analysis from the FTSE 100 index

\begin{tabular}{|c|l|l|l|}
\hline Number & Business type & Company & Abbreviation \\
\hline \hline 1 & Mining & Anglo American plc & AAL \\
2 & & BHP Billiton & BHP \\
\hline 3 & Energy (oil/gas) & BG Group & BG \\
4 & & BP & BP \\
5 & & Royal Dutch Shell & RDSB \\
\hline 6 & Energy (distribution) & Centrica & CNA \\
7 & & National Grid & NG \\
\hline 8 & Finance (bank) & Barclays plc & BARC \\
9 & & HBOS & HBOS \\
10 & & HSBC HLDG & HSBC \\
11 & & Lloyds & LLOY \\
\hline 12 & Finance (insurance) & Admiral & ADM \\
13 & & Aviva & AV \\
14 & & LandSecurities & LAND \\
15 & & Prudential & PRU \\
16 & & Standard Chartered & STAN \\
\hline 17 & Food production & Unilever & ULVR \\
\hline 18 & Consumer & Diageo & DGE \\
19 & goods/food/drinks & SABMiller & SAB \\
20 & & TESCO & TSCO \\
\hline 21 & Tobacco & British American Tobacco & BATS \\
22 & & Imperial Tobacco & IMT \\
\hline 23 & Pharmaceuticals & AstraZeneca & AZN \\
24 & (inc. research) & GlaxoSmithKline & GSK \\
\hline 25 & Telecommunications & BT Group & BTA \\
26 & & Vodafone & VOD \\
\hline 27 & Travel/leasure & Compass Group & CPG \\
\hline 28 & Media (broadcasting) & British Sky Broadcasting & BSY \\
\hline 29 & Aerospace/ & BAE System & BA \\
30 & defence & Rolls-Royce & RR \\
\hline \hline & & & \\
\hline \hline
\end{tabular}

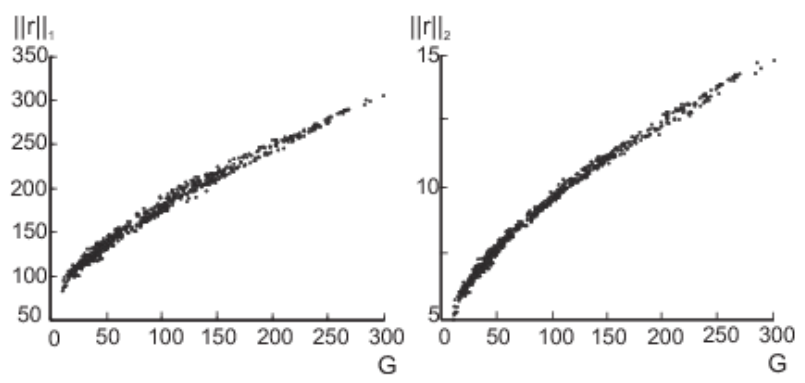

Figure 9: Scatter diagrams for two pairs of indicators: $G-\|r\|_{1}, G-\|r\|_{2}$, 

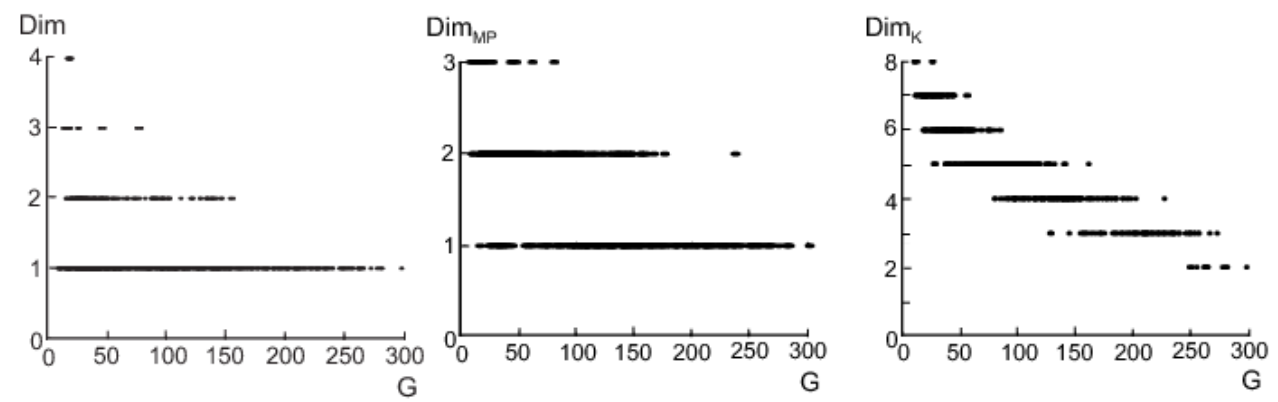

Figure 10: Scatter diagrams for three pairs of indicators: $G-\operatorname{Dim}, G-\operatorname{Dim}_{\mathrm{MP}}, G-\mathrm{Dim}_{\mathrm{K}}$, where Dim is amount of principal components estimated due to the broken stick model, $\operatorname{Dim}_{\mathrm{MP}}$ is an estimate due to the random matrix theory (2.4), and $\operatorname{Dim}_{\mathrm{K}}$ is the amount of principal components estimated by Kaiser's rule $\lambda_{i}>1$.
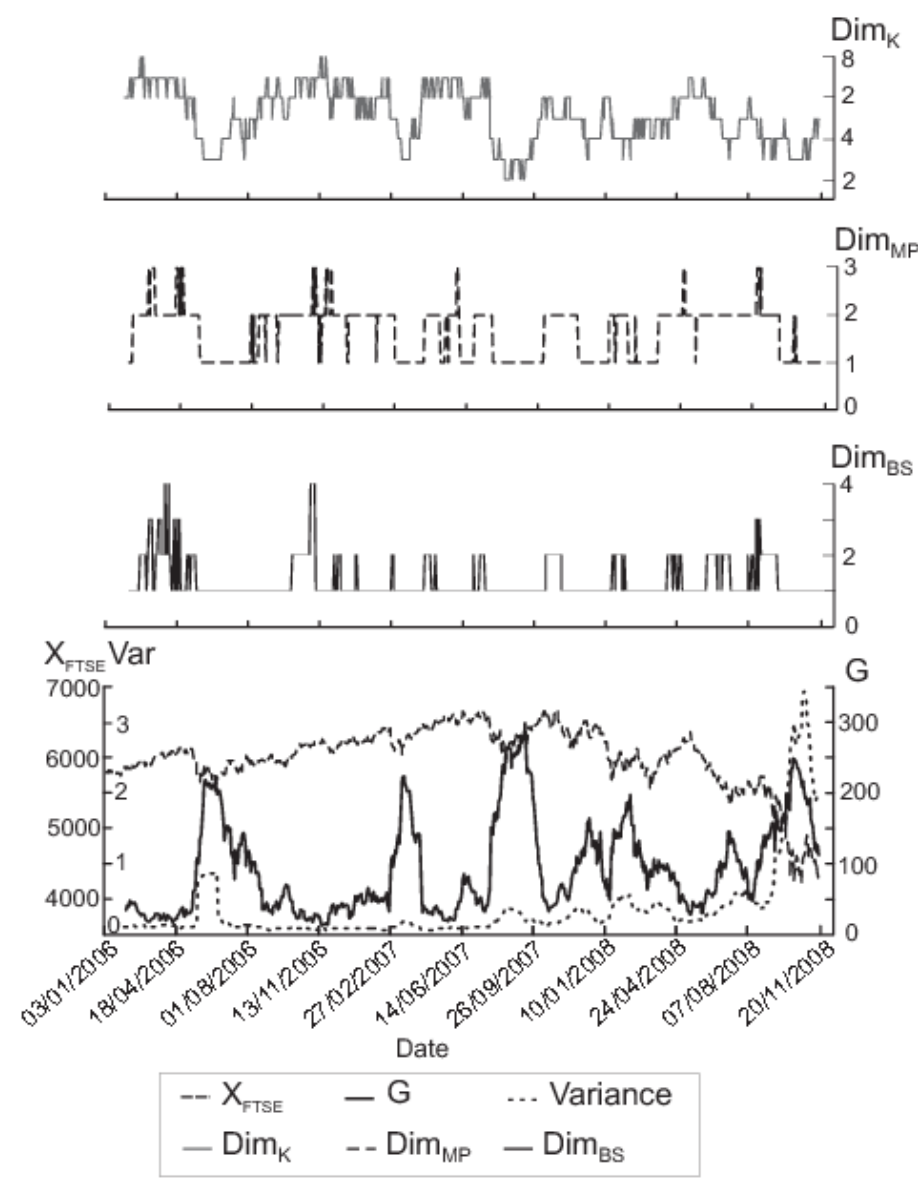

Figure 11: Dynamics of FTSE index, G, Variance, and Dimensions $\operatorname{Dim}_{\mathrm{MP}}$, $\operatorname{Dim}_{\mathrm{MP}}, \mathrm{Dim}_{\mathrm{K}}$ estimated due to the various models. 
a)

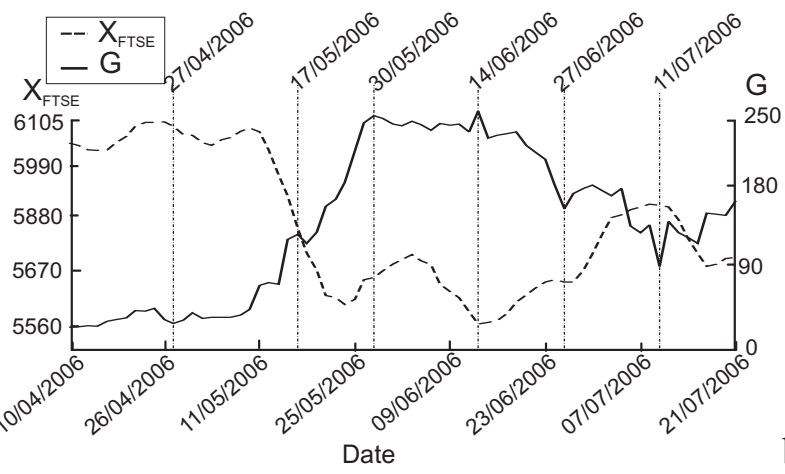

b)

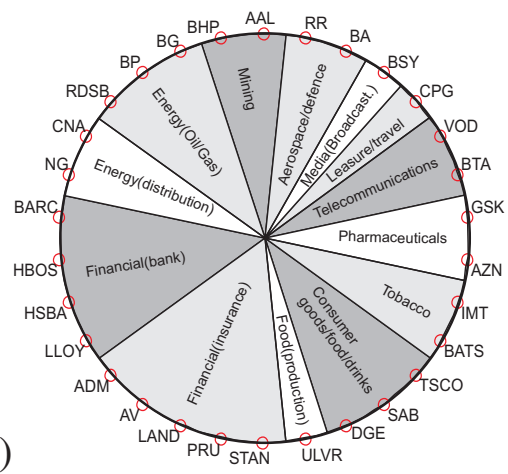

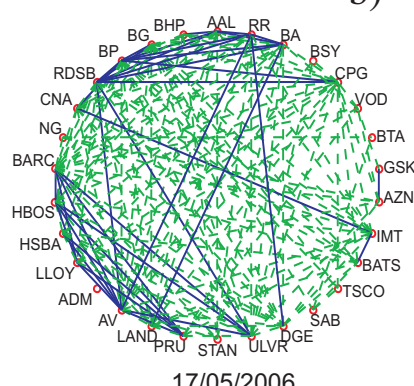

$17 / 05 / 2006$

c)

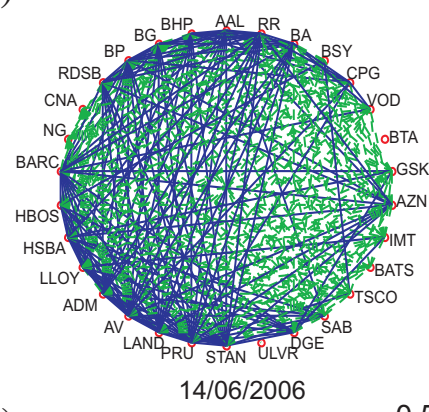

d)

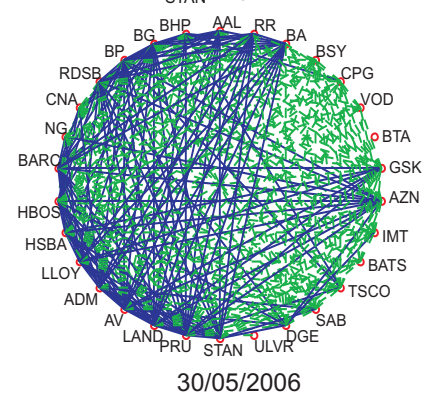

30/05/2006

$0.5<\left|r_{i j}\right|<0.7$

$\left|r_{i j}\right| \geqslant 0.7$

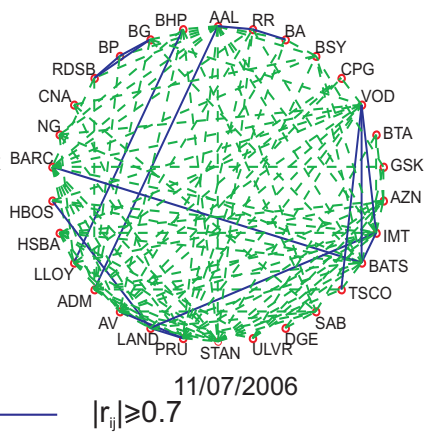

Figure 12: Correlation graphs for six positions of sliding time window on interval 10/04/2006 21/07/2006. a) Dynamics of FTSE100 (dashed line) and of $G$ (solid line) over the interval, vertical lines correspond to the points that were used for the correlation graphs. b) Thirty companies for analysis and their distributions over various sectors of economics. c) The correlation graphs for the first three points, FTSE100 decreases, the correlation graph becomes more connective. d) The correlation graphs for the last three points, FTSE100 increases, the correlation graph becomes less connective. 
a)

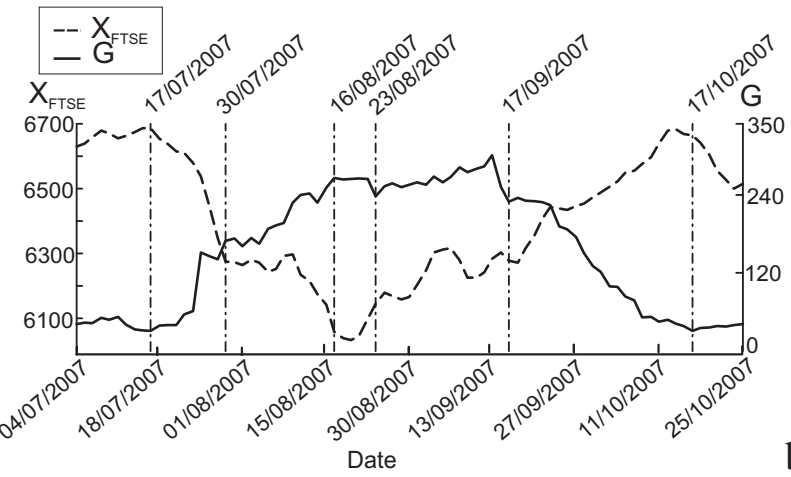

b)
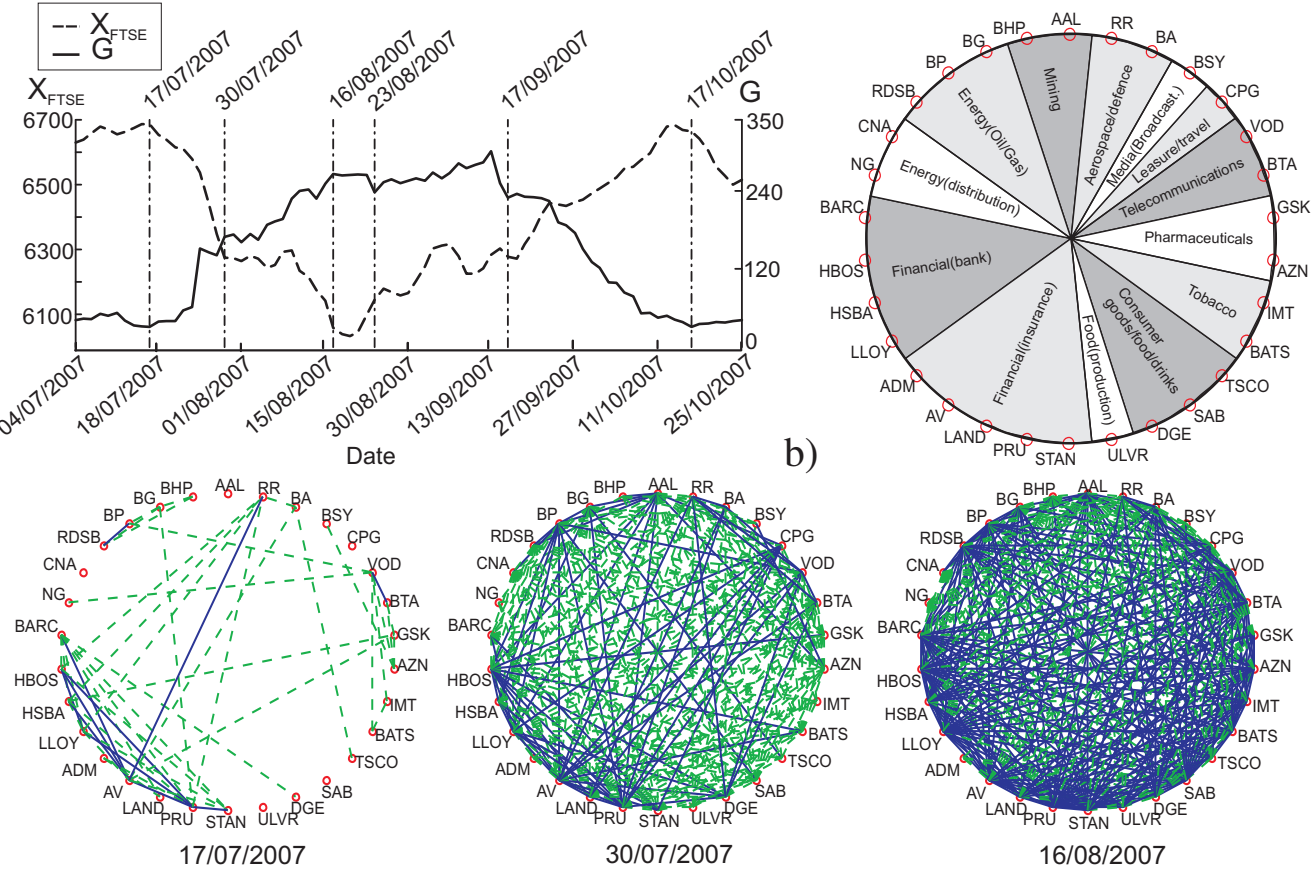

c)

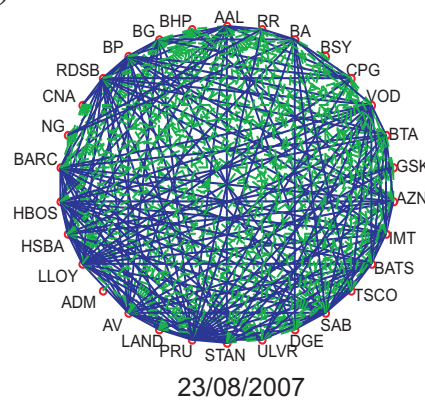

d)

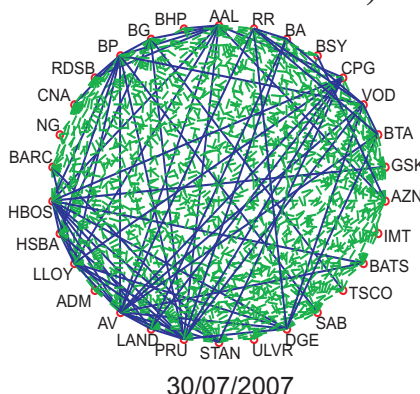

$0.5<\left|r_{i j}\right|<0.7$

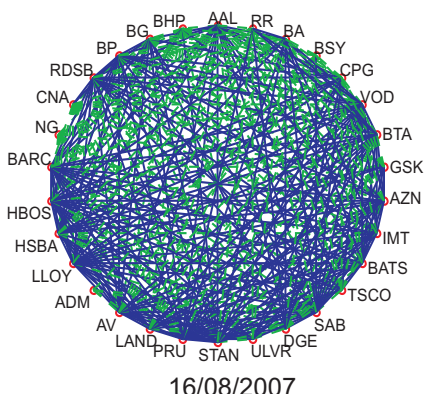

$\left|r_{i j}\right| \geqslant 0.7$

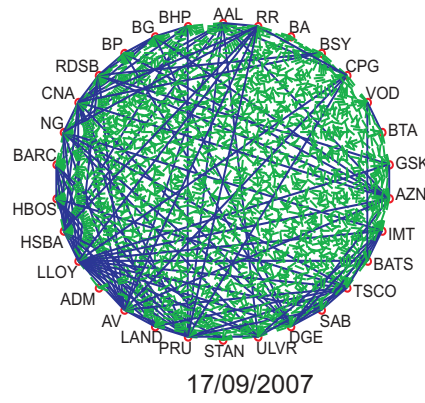

$0.5<\left|r_{\mathrm{i} j}\right|<0.7$

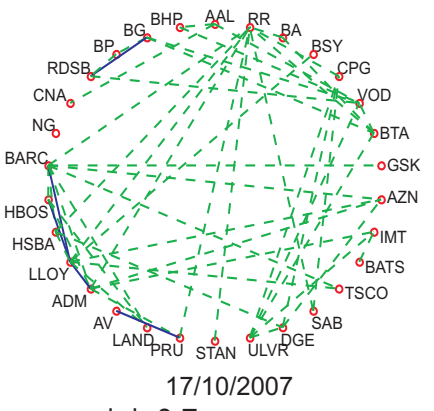

$\left|r_{i j}\right| \geqslant 0.7$

Figure 13: Correlation graphs for six positions of sliding time window on interval 04/07/2007 25/10/2007. a) Dynamics of FTSE100 (dashed line) and of $G$ (solid line) over the interval, vertical lines correspond to the points that were used for the correlation graphs. b) Thirty companies for analysis and their distributions over various sectors of economics. c) The correlation graphs for the first three points, FTSE100 decreases, the correlation graph becomes more connective. d) The correlation graphs for the last three points, FTSE100 increases, the correlation graph becomes less connective. 
a)

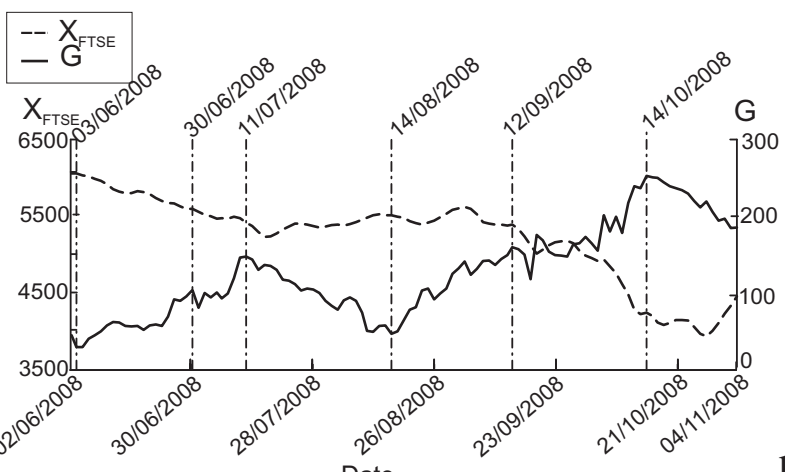

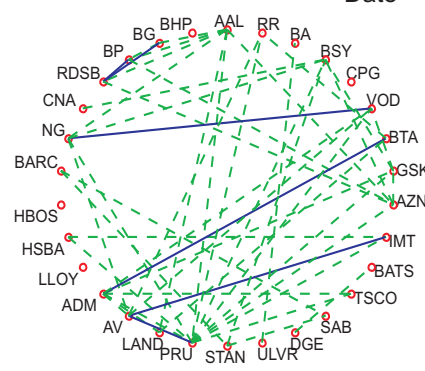

03/06/2008

c)

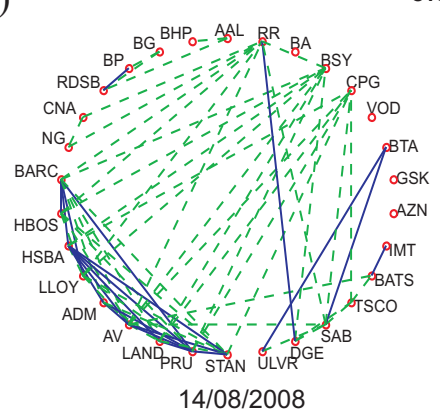

d)

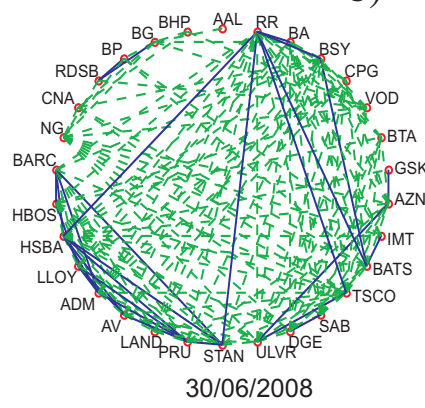

b)
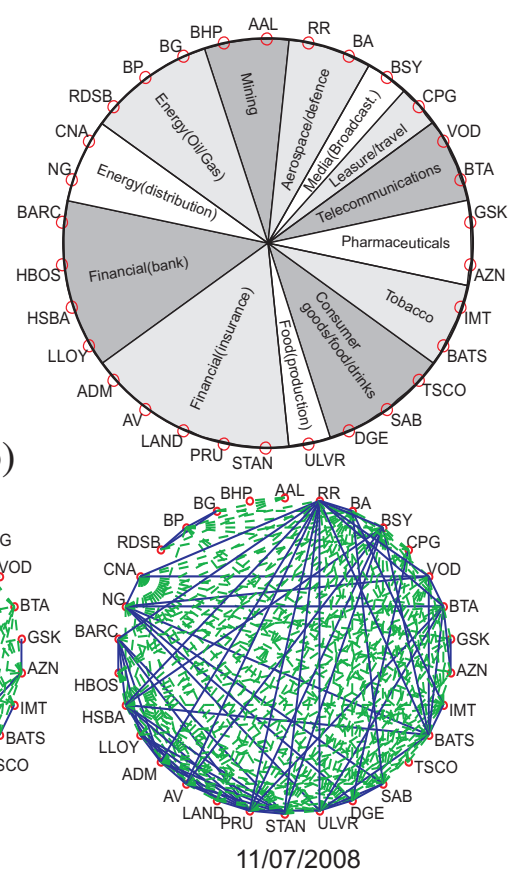

$\left|r_{i j}\right| \geqslant 0.7$

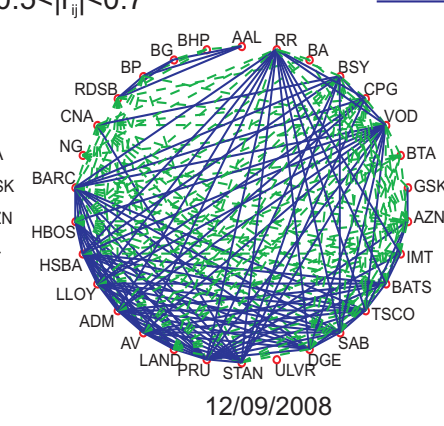

$0.5<\left|r_{i j}\right|<0.7$

Figure 14: Correlation graphs for six positions of sliding time window on interval 02/06/2008 04/11/2008. a) Dynamics of FTSE100 (dashed line) and of $G$ (solid line) over the interval, vertical lines correspond to the points that were used for the correlation graphs. b) Thirty companies for analysis and their distributions over various sectors of economics. c) The correlation graphs for the first three points, FTSE100 decreases, the correlation graph becomes more connective. Between the third and the 4th points FTSE100 increased, and the first graph here is more rarefied than at the third point. Between the third and the 4th points FTSE100 slightly increased, correlation decreased, and the first graph at the next row is more rarefied than at the third point. d) The correlation graphs for the last three points, FTSE100 decreases, the correlation graph becomes more connective. 
$04 / 11 / 2008$, is a part of the recent financial crisis. In each interval we selected six points and analyzed the structure of correlations for each of these points (for a time window, which precedes this point). For each selected point, we create a correlation graph, solid lines represent correlation coefficient $\left|r_{i j}\right| \geq \sqrt{0.5}(\sqrt{0.5}=\cos (\pi / 4) \approx 0.707)$, dashed lines represent correlation coefficient $\sqrt{0.5}>\left|r_{i j}\right| \geq 0.5$ : Figs. 12c,d, 13c,d, 14c,d. On these correlation graphs it is easy to observe, how critical correlations appear, how are they distributed between different sectors of economics, and how the crisis moves from one sector to another.

There is no symmetry between the periods of the FTSE index decrease and recovering. For example, in Fig. 12c we see that at the beginning (falling down) the correlations inside the financial sector are important and some correlations inside industry are also high, but in the corresponding recovering period (Fig. 12d) the correlations between industry and financial institutions become more important.

All the indicators demonstrate the most interesting behavior at the end of 2008 (Fig. 11). The grows of variance in the last peak is extremely high, but the increase of correlations is rather modest. If we follow the logic of the basic hypothesis (Fig. 1), then we should suspect that the system is going to "the other side of crisis", not to recovery, but to disadaptation, this may be the most dangerous symptom.

\subsubsection{Graphs for Correlations in Time}

The vector of attributes that represents a company is a 20 day fragment of the time series. In standard biophysical research, we studied correlations between attributes of an individual, and rarely, correlation between individuals for different attributes. In econophysics the standard situation is opposite. Correlation in time is evidenced to be less than correlation between companies [25]. Nevertheless, correlation between days in a given time window may be a good indicator of crisis.

Let us use here $G_{T}$ for the weight of the correlation graph in time. Because correlation in time is less than between stocks, we select here another threshold: $G_{T}$ is the sum of the correlation coefficients with absolute value greater then 0.25, Dynamics FTSE together with values of $G_{T}$ are presented in Fig. 15.

In Figs. 16, 17, 18 we combined graphs of days correlations - 20 trading days prior to the selected days. In these graphs, solid lines represent correlation coefficient $\left|r_{i j}\right| \geq 0.5$, dashed lines represent correlation coefficient $0.5>\left|r_{i j}\right| \geq 0.25$.

Analysis of dynamics of $G_{T}$ allows us to formulate a hypothesis: typically, after the increase of $G_{T}$ the decrease of FTSE100 index follows (and, the decrease of $G_{T}$ preseeds the increase of FTSE100). The time delay is approximately two working weeks. In that sense, the correlation in time seems to be better indicator of the future change than the correlation between stocks which has not such a time gap. On the other hand, the amplitude of change of $G_{T}$ is much smaller, and some of decreases of FTSE100 index could not be predicted by increases of $G_{T}$ (Fig. 15).

These observations are still preliminary and need future analysis for different financial time series.

Strong correlation between days appears also with some time gap: the links emerge not obligatory between nearest days, but mostly with interval 4-15 days (see Figs. 16, 18). 


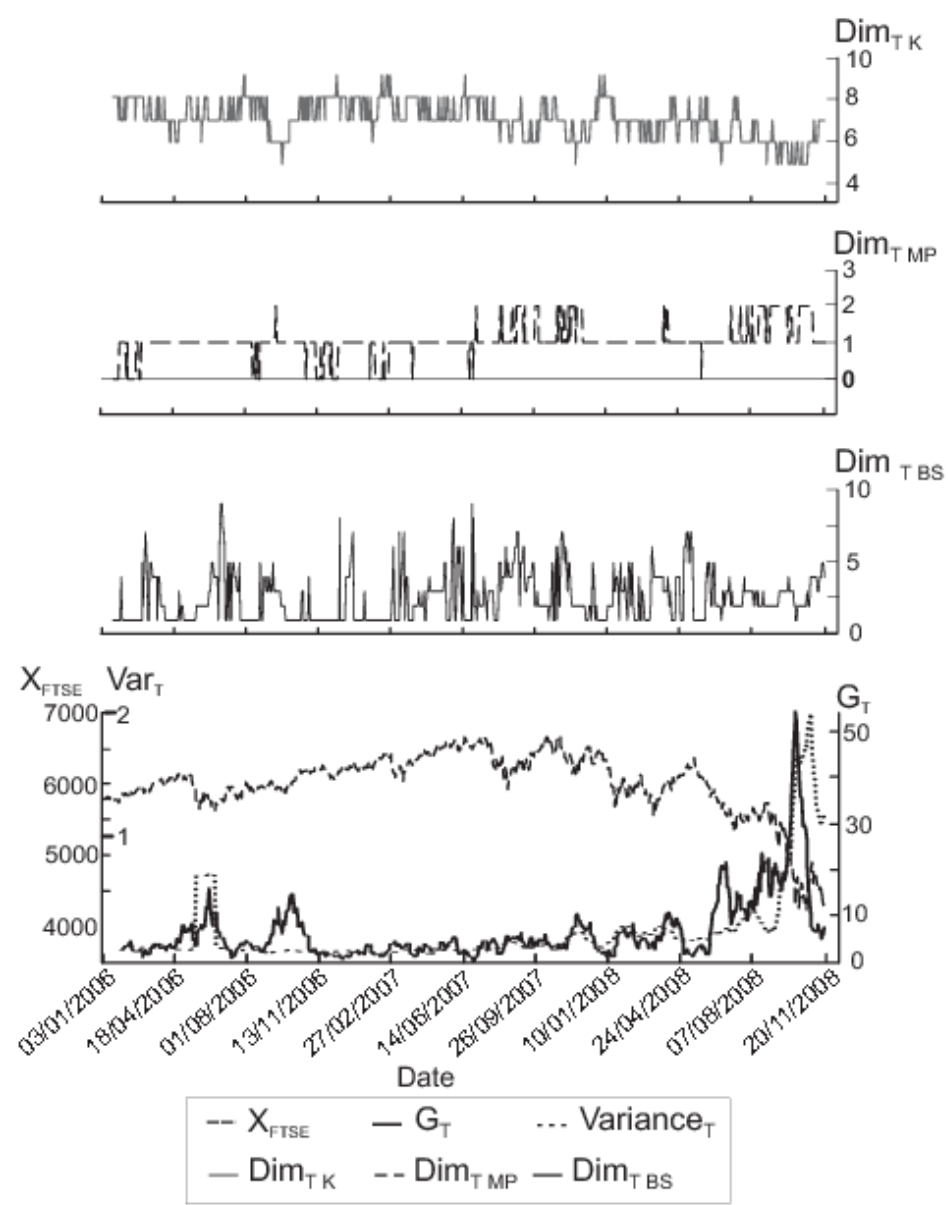

Figure 15: Dynamics of the market $X_{F T S E}$, weight of the correlation graph in time $G_{T}$ (the sum of the correlation coefficients with absolute value greater then 0.25 ), Variance (volatility), and Dimensions Dim DP $_{\text {, Dim }}$, Dim $\mathrm{K}$ estimated due to the various models for the correlation matrix in time. 


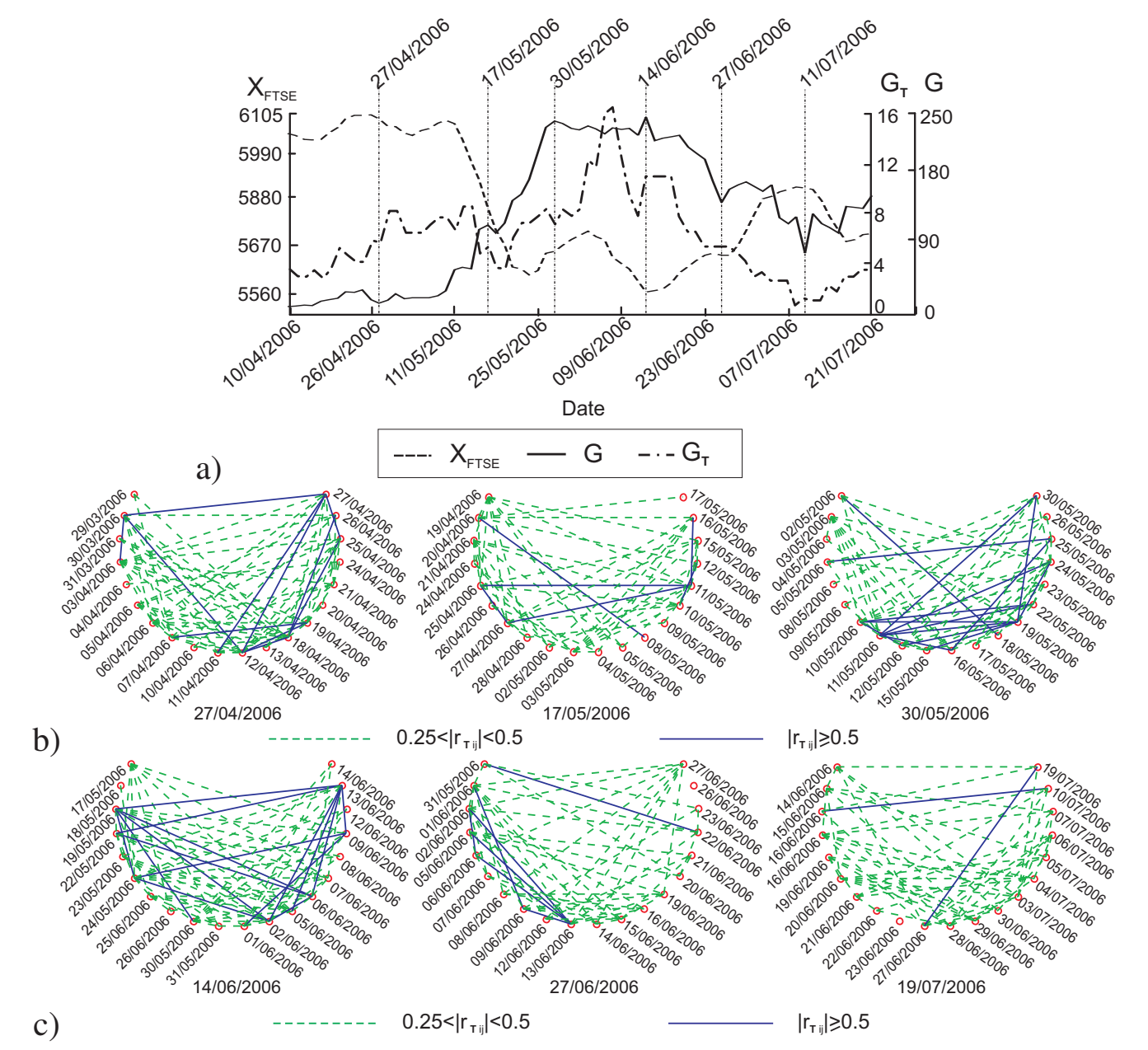

Figure 16: Graphs for correlation in time for six positions of sliding time window on interval 10/04/2006 - 21/07/2006. a) Dynamics of FTSE100 (dashed line), $G$ (solid line) and $G_{T}$ (dash-anddot line) over the interval, vertical lines correspond to the points that were used for the correlation graphs. b) The correlation graphs for the first three points: FTSE100 decreases and the correlation graph becomes more connective. c) The correlation graphs for the last three points: FTSE100 increases and the correlation graph becomes less connective. 


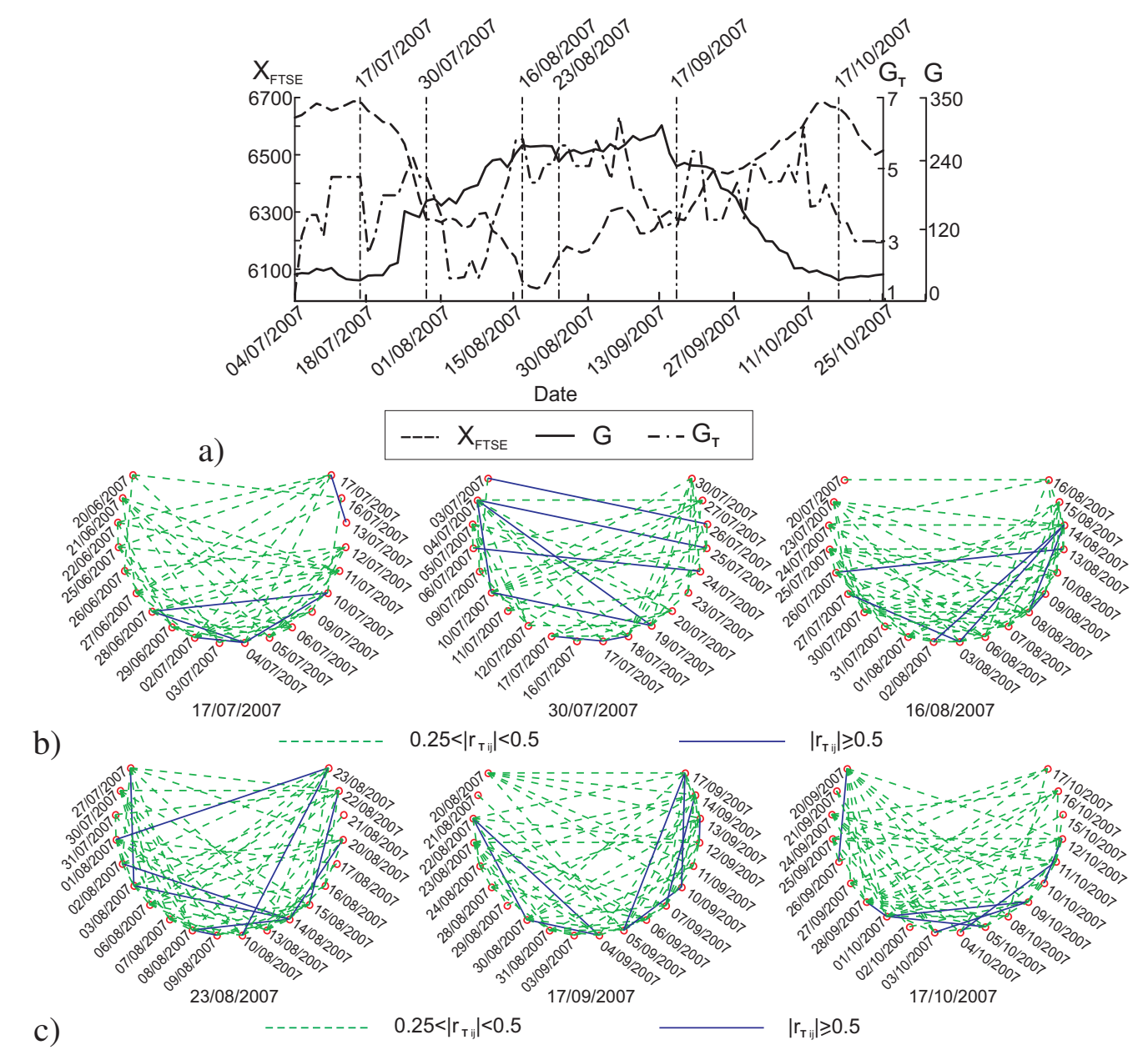

Figure 17: Graphs for correlation in time for six positions of sliding time window on interval 04/07/2007 - 25/10/2007. a) Dynamics of FTSE100 (dashed line), $G$ (solid line) and $G_{T}$ (dash-anddot line) over the interval, vertical lines correspond to the points that were used for the correlation graphs. b) The correlation graphs for the first three points: FTSE100 decreases and the correlation graph becomes more connective. c) The correlation graphs for the last three points: FTSE100 increases and the correlation graph becomes less connective. 

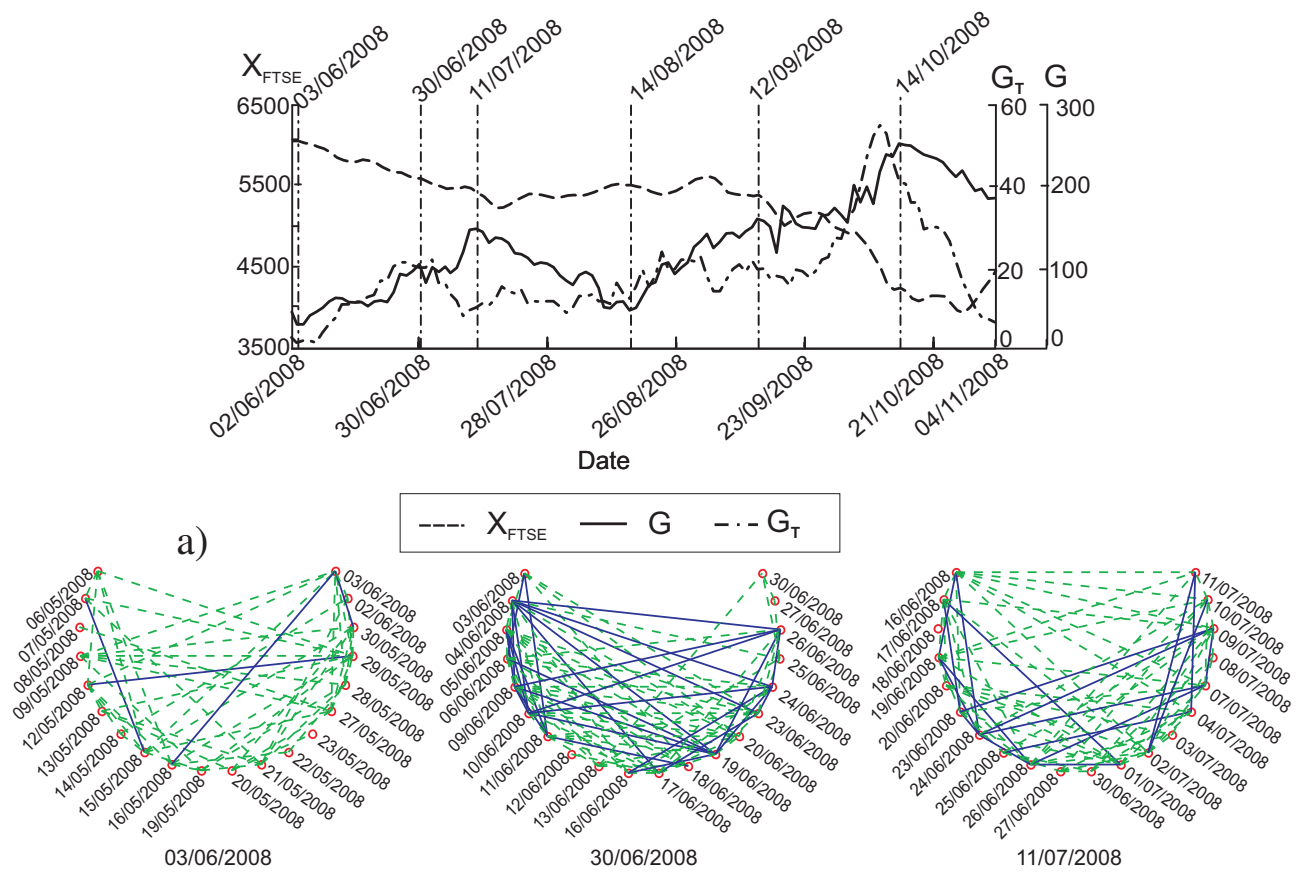

b)

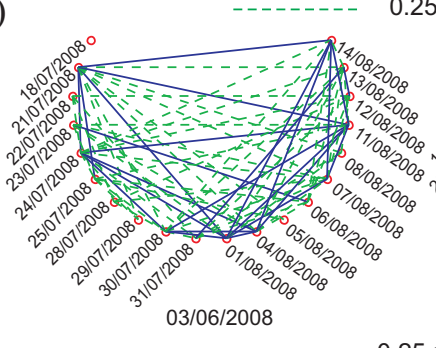

$$
0.25<\left|r_{T i j}\right|<0.5
$$
$\left|\mathrm{r}_{\mathrm{Ti}}\right| \geqslant 0.5$

c)
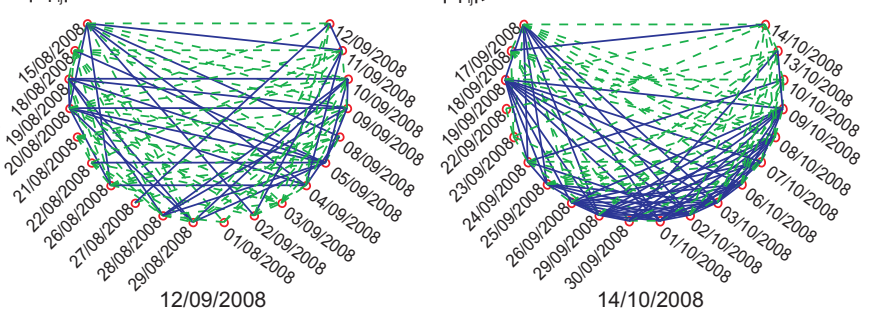
$\left|\mathrm{r}_{\mathrm{T} i \mathrm{i}}\right| \geqslant 0.5$

Figure 18: Correlation graphs for six positions of sliding time window on interval 02/06/2008 04/11/2008. a) Dynamics of FTSE100 (dashed line), $G$ (solid line) and $G_{T}$ (dash-and-dot line) over the interval, vertical lines correspond to the points that were used for the correlation graphs. b) Thirty companies for analysis and their distributions over various sectors of economics. c) The correlation graphs for the first three points: FTSE100 decreases and the correlation graph becomes more connective. Between the third and the 4th points FTSE100 increases, and the first graph here is more rarefied than at the third point. Between the third and the 4th points FTSE100 slightly increases, correlation decreased, and the first graph at the next row is more rarefied than at the third point. d) The correlation graphs for the last three points: FTSE100 decreases and the correlation graph becomes more connective. 


\subsection{Correlations and Crisis in Financial Time Series}

In economics and finance, the correlation matrix is very important for the practical problem of portfolio optimization and minimization of risk. Hence, an important problem arises: are correlations constant or not? The hypothesis about constant correlations was tested for monthly excess returns for seven countries (Germany, France, UK, Sweden, Japan, Canada, and US) over the period 1960-90 [27]. Correlation matrices were calculated over a sliding window of five years. The inclusion of October 1987 in the window led to increase of correlation in that window. After analysis of correlations in six periods of five years the null hypothesis of a constant correlation matrix was rejected. In addition, the conditional correlation matrix was studied. The multivariate process for asset return was presented as

$$
R_{t}=m_{t-1}+e_{t} ; \quad m_{t-1}=\mathbf{E}\left(R_{t} \mid F_{t-1}\right),
$$

where $R_{t}$ is a vector of asset returns and $m_{t-1}$ is the vector of expected returns at time $t$ conditioned on the information set $F_{t-1}$ from the previous step. Vector $e_{t}$ is the unexpected (unpredicted) component in the asset returns. Correlations between its components are called conditional correlations. It was demonstrated that these conditional correlations are also not constant. Two types of change were found. Firstly, the correlations have statistically significant time trend and grow in time. The average increase in correlation over 30 years is 0.36 . Secondly, correlations in periods of high volatility (high variance) are higher. To obtain this result, the following model for the correlation coefficient was identified:

$$
r_{t}^{i, \mathrm{us}}=r_{0}^{i, \mathrm{us}}+r_{1}^{i, \mathrm{us}} S_{t}^{\mathrm{us}}
$$

where $r_{t}^{i \text {,us }}$ is the correlation coefficient between the unexpected (unpredicted) components in the asset returns for the $i$ th country and the US, $S_{t}$ is a dummy variable that takes the value 1 if the estimated conditional variance of the US market for time $t$ is greater than its unconditional (mean) value and 0 otherwise. The estimated coefficient $r_{1}$ is positive for all countries. The average over all countries for $r_{0}$ is equal to 0.430 , while the average turbulence effect $r_{1}$ is 0.117 [27]. Finally, it was demonstrated that other informational variables can explain more changes in correlations than just the "high volatility - low volatility" binning.

To analyze correlations between European equity markets before and after October 1987, three 76-month periods were compared: February 1975-May 1981, June 1981-September 1987, and November 1987-February 1994 [35]. The average correlation coefficient for 13 equity markets (Europe + US) increased from 0.37 in June 1981-September 1987 to 0.5 in November 1987February 1994. The amount of significant principal components selected by Kaiser's rule decreases from 3 (in both periods before October 1987) to 2 (in the period after October 1987) for all markets and even from 3 to 1 for 12 European markets [35]. Of course, in average values for so long periods it is impossible to distinguish the consequences of the October 1987 catastrophe and a trend of correlation coefficients (that is, presumably, nonlinear).

Non-stationarity of the correlation matrix was demonstrated in a detailed study of the financial empirical correlation matrix of the 30 companies which Deutsche Aktienindex (DAX) comprised during the period 1988-1999 [11]. The time interval (time window) is set to 30 and continuously 
moved over the whole period. It was evidenced that the drawups and the drawdowns of the global index (DAX) are governed, respectively, by dynamics of significantly distinct nature. The drawdowns are dominated by one strongly collective eigenstate with a large eigenvalue. The opposite applies to drawups: the largest eigenvalue moves down which is compensated by a simultaneous elevation of lower eigenvalues. Distribution of correlation coefficients for these data have a distinctive bell-like shape both for one time window (inside one correlation matrix) and for ensemble of such sliding windows in a long time period.

This observation supports the idea of applying the theory of the Gaussian matrix ensembles to analysis of financial time series. The random matrix theory gives a framework for analysis of the cross-correlation matrix for multidimensional time series. In that framework, stock price changes of the largest 1000 U.S. companies were analyzed for the 2-year period 1994-1995 [17], and statistics of several largest eigenvalues was evidenced to be far from the random matrix prediction, but the distribution of "the rest" of eigenvalues and eigenvectors satisfies the random matrix ensemble. The crucial question is: where is the border between the random and the non-random parts of spectra? Formula (2.4) gives in this case $\lambda_{\max } \approx 2$. The random matrix theory predicts for the Gaussian orthogonal ensembles that the components of the normalized eigenvectors are distributed according to a Gaussian probability distribution with mean zero and variance one. Eigenvectors corresponding to most eigenvalues in the "bulk" $(\lambda<2)$ have the Gaussian distribution, but eigenvectors with bigger eigenvalues significantly deviate from this. [17].

This kind of analysis was continued for the three major US stock exchanges, namely the New York Stock Exchange (NYSE), the American Stock Exchange (AMEX), and the National Association of Securities Dealers Automated Quotation (NASDAQ) [42]. The concept of "deviating eigenvectors" was developed, these vectors correspond to the eigenvalues which are systematically outside the random matrices ensembles predictions. Analysis of "deviating eigenvectors" which are outside the random matrices ensembles predictions (2.4) gives information of major factors common to all stocks, or to large business sectors. The largest eigenvalue was identified as the "market mode". During periods of high market volatility values of the largest eigenvalue are large. This fact was commented as a strong collective behavior in regimes of high volatility. For the largest eigenvalue, the distribution of coordinates of the eigenvector has very remarkable properties:

- It is much more uniform than the prediction of the random matrix theory (authors of Ref. [42] described this vector as "approximately uniform", suggesting that all stocks participate in this "market mode");

- Almost all components of that eigenvector have the same sign.

- A large degree of cross correlations between stocks can be attributed to the influence of the largest eigenvalue and its corresponding eigenvector

Two interpretations of this eigenvector were proposed: it corresponds either to the common strong factor that affects all stocks, or it represents the "collective response" of the entire market to stimuli.

Spectral properties of the correlation matrix were analyzed also for 206 stocks traded in Istanbul Stock Exchange Market during the 5-year period 2000-2005 [8]. One of the main results of 
this research is the observation that the correlations among stocks are mostly positive and tend to increase during crises. The number of significant eigenvalues (outside the random matrix interval) is smaller than it was found in previous study of the well-developed international market in the US. The possible interpretation is: the emerging market is ruling by smaller amount of factors.

Increase of correlations in crisis time was demonstrated by analysis of 150 years of market dynamics [15]. As a result, in the year 2004 it was mentioned very optimistically: "Our tests suggest that the structure of global correlations shifts considerably through time. It is currently near an historical high - approaching levels of correlation last experienced during the Great Depression". Nevertheless, it remains unclear, does the correlation cause the transmission chain of collapse or is it inextricably tied to it [56]?

There are several types of explanation of these correlation effects. One can look for the specific reasons in the balance between specialization and globalization, in specific fiscal, monetary, legal, cultural or even language conditions, in dynamics of fundamental economic variables such as interest rates and dividend yields, in the portfolio optimization by investors, and in many similar more or less important processes. These specific explanations could work, but for the effect of such a generality it is desirable to find a theory of compatible generality. Now we can mention three sources for such a theory:

1. Theory of individual adaptation of similar individuals to a similar system of factors;

2. Theory of interaction: information interaction, co-ordination, or deeper integration;

3. Theory of collective effects in market dynamics.

The first approach (supported by biological data) is a sort of mean-field theory: everybody is adapting to a field of common factors, and altogether change the state of that system. There are two types of argumentation here: similarity of factors, or similarity of adaptation mechanisms (or both):

- In the period of crisis there appear the same challenges for most of the market participants, and correlation increases because they have to answer the same challenge and struggle with the same factors.

- In the period of crisis all participants are under pressure. The nature of that pressure may be different, but the mobilization mechanisms are mostly universal. Similar attempts of adaptation produce correlation as a consequence of crisis.

This theory is focused on the adaptation process, but may be included into any theory of economical dynamic as an adaptation feedback. We study the adaptation of individuals in the "mean field", and consider dynamics of this field as external conditions.

The interaction theory may be much more reach (and complicated). For example, it can consider such an effect of behavior in crisis: there is a lack of information and of known optimal solutions, therefore, different agents try to find clues to rational behavior in the behavior of other agents, and the correlation increase. Coordination in management and in financial politics is the 
obvious effect of interaction too, and we can observe also a deeper integration, which causes fluxes of moneys and goods.

Collective effects in market dynamics may also generate correlations and, on the other hand, can interact with correlations which appear by any specific or nonspecific reasons. For example, high levels of correlation often lead to the loss of dissipation in dynamics and may cause instability.

Further in this work, we focus on the theory of individual adaptation of similar individuals to a similar system of factors. We accept the hypothesis that this component is common for ecological physiology of adaptation to hard living conditions, and for economics in crisis. This theory does not pretend to explain all possible reasons for correlation in crisis. Other effects may be important, and could be incorporated later, if necessary.

\section{Theoretical approaches}

\subsection{The "Energy of Adaptation" and Factors-Resources Models}

\subsubsection{Factors and Systems}

We start from a very simple abstract that is close to the usual factor analysis. We consider several systems that are under influence of several factors $F_{1}, \ldots F_{q}$. Each factor has its intensity $f_{i}(i=$ $1, \ldots q)$. For convenience, we consider all these factors as negative or harmful (later, after we introduce fitness $W$, it will mean that all partial derivatives are non-positive $\partial W / \partial f_{i} \leq 0$ ). This is just a convention about choice of axes directions: a wholesome factor is just a "minus harmful" factor.

Each system has its adaptation systems, a "shield" that can decrease the influence of these factors. In the simplest case, it means that each system has an available adaptation resource, $R$, which can be distributed for neutralization of factors: instead of factor intensities $f_{i}$ the system is under pressure from factor values $f_{i}-a_{i} r_{i}$ (where $a_{i}>0$ is the coefficient of efficiency of factor $F_{i}$ neutralization by the adaptation system and $r_{i}$ is the share of the adaptation resource assigned for the neutralization of factor $F_{i}, \sum_{i} r_{i} \leq R$ ). The zero value $f_{i}-a_{i} r_{i}=0$ is optimal (the fully compensated factor), and the further compensation is impossible and senseless.

Interaction of each system with a factor $F_{i}$ is described by two quantities: the factor $F_{i}$ pressure $\psi_{i}=f_{i}-a_{i} r_{i}$ and the resource assigned to the factor $F_{i}$ neutralization. The first quantity characterizes, how big the uncompensated harm is from that factor, the second quantity measures, how intensive is the adaptation answer to the factor (or how far the system was modified to answer the factor $F_{i}$ pressure).

The question about interaction of various factors is very important, but, first of all, let us study the one-factor models. 


\subsubsection{One-Factor Model}

Tension-Driven Models. In these models, observable properties of interest $x_{k}(k=1, \ldots m)$ can be modeled as functions of factor pressure $\psi$ plus some noise $\epsilon_{k}$.

Let us consider one-factor systems and linear functions (the simplest case). For the

$$
x_{k}=\mu_{k}+l_{k} \psi+\epsilon_{k},
$$

where $\mu_{k}$ is the mean value of $x_{k}$ for fully compensated factor, $l_{k}$ is a coefficient, $\psi=f-a r_{f}$, and $r_{f} \leq R$ is amount of available resource assigned for the factor neutralization. The values of $\mu_{k}$ could be considered as "normal" (in the sense opposite to "pathology"), and noise $\epsilon_{k}$ reflects variability of norm.

If systems compensate as much of factor value, as it is possible, then $r_{f}=\min \{R, f / a\}$, and we can write:

$$
\psi=\left\{\begin{array}{l}
f-a R, \text { if } f>a R \\
0, \text { else. }
\end{array}\right.
$$

Individual systems may be different by the value of factor intensity (the local intensity variability), by amount of available resource $R$ and, of course, by the random values of $\epsilon_{k}$. If all systems have enough resource for the factor neutralization $(a r>f)$ then all the difference between them is in the noise variables $\epsilon_{k}$.

If the factor value increases, and for some of the systems the factor intensity $f$ exceeds the available compensation $a R$ then for these systems $\psi>0$ and the term $l_{k} \psi$ in Eq. (5.1) becomes important. If the noise of the norm $\epsilon_{k}$ is independent of $\psi$ then the correlation between different $x_{k}$ increases monotonically with $f$.

With increase of the factor intensity $f$ the dominant eigenvector of the correlation matrix between $x_{k}$ becomes more uniform in the coordinates, which tend asymptotically to $\pm \frac{1}{\sqrt{m}}$.

Correlation between systems also increases (just transpose the data matrix), and coordinates of the dominant eigenvector similarly tend to values $\frac{1}{\sqrt{n}}$ (which are positive), but this tendency has character of a "wave of the resource exhausting" which spreads through the systems following the rule (5.2).

The observation of Ref. [42] partially supports the uniformity of the eigenvector that corresponds to the largest eigenvalue which "represents the influence of the entire market that is common to all stocks." Fig. 8d from Ref. [42] shows that the components of this eigenvector are positive and "almost all stocks participate in the largest eigenvector." Also, in Ref. [11] it was demonstrated that in the periods of drawdowns of the global index (DAX) there appears one strongly dominant eigenvalue for synchronous correlations between 30 companies from DAX. Similar results for 30 British companies are presented in Figs. 15, 11. In physiology, we also found these "maximum integration" effects for various loads on organisms. When the pressure is lower then, instead of one dominant eigenvector which represents all functional systems of an organism, there appears a group of eigenvectors with relatively high eigenvalues. Each of these vectors has significant components for attributes of a specific group of functional systems, and the intersection of those groups for different eigenvectors is not large. In addition, the effect of factors "disintegration" because of overload was also observed. 
This one-factor tension-driven model could be extracted from the Selye study of "adaptation energy" $[51,52]$. To prove the existence of such a hidden resource variable he used very similar reasoning (without formulas). We propose to name this model the Selye model.

Response-Driven Models. What is more important for values of the observable quantities $x_{k}$ : the current pressure of the factors, or the adaptation to this factor which modified some of parameters? Perhaps, both, but let us introduce now the second simplest model.

In the response-driven model of adaptation, the quantities $x_{k}$ are modeled as functions of adaptive response $a r_{f}$ plus some noise $\epsilon_{k}$ :

$$
x_{k}=\mu_{k}+q_{k} a r_{f}+\epsilon_{k} .
$$

When $f$ increases then, after threshold $f=a R$, the term $l_{k} a r_{f}$ transforms into $l_{k} a R$ and does not change further. The observable quantities $x_{k}$ are not sensitive to changes in the factor intensity $f$ when $f$ is sufficiently large. This is the significant difference from the behavior of the tensiondriven model (5.1), which is not sensitive to change of $f$ when $f$ is sufficiently small.

Tension-and-Response Driven 2D One-Factor Models. This model is just a linear combination of Eqs. (5.1) and (5.3)

$$
x_{k}=\mu_{k}+l_{k} \psi+q_{k} a r_{f}+\epsilon_{k} .
$$

For small $f$ (comfort zone) $\psi=0$, the term $l_{k} \psi$ vanishes, $a r_{f}=f$ and the model has the form $x_{k}=\mu_{k}+q_{k} f+\epsilon_{k}$. For intermediate level of $f$, if systems with both signs of inequality $f \gtreqless a R$ are present, the model imitates $2 \mathrm{D}$ (two-factor) behavior. After the threshold $f \geq a R$ is passed for all systems, the model demonstrates $1 \mathrm{D}$ behavior again: $x_{k}=\mu_{k}+l_{k} f+\left(q_{k}-l_{k}\right) a R+\epsilon_{k}$. For small $f$ the motion under change of $f$ goes along direction $q_{k}$, for large $f$ it goes along direction $l_{k}$.

Remark About One-Factor Econometrics Time Series Models. In this Sec., we consider models with adaptation to the pressure of one factor. It is necessary to distinguish these models from the classical one-factor econometrics models which assume that the returns of stocks $\left(\rho_{i}\right)$ are controlled by one factor, the "market" return $M(t)$. In this model, for any stock

$$
\rho_{i}(t)=a_{i}+b_{i} M(t)+\epsilon_{i}(t)
$$

where $\rho_{i}(t)$ is the return of the $i$ th stock at time $t, a_{i}$ and $b_{i}$ are real parameters, and $\epsilon_{i}(t)$ is a zero mean noise. In our models, the factor pressure characterizes the time window and is slower variable than the return. Return values at different time moments are attributes $\left(x_{k}\right)$, the vector of these parameters characterizes a system, and $k$ stands for time moment. Let us compare this model to Eq. (5.1). We find that $b_{i}$ stands for $\psi$ value for an individual ( $i$ th) system and vector of market values $M(t)$ in a given time window plays role of vector $l_{k}$ from Eq. (5.1). Factor pressure is not constant in time and is just approximate constant in the time window. This makes the considered 
class of adaptation models significantly wider than the simplest one-factor econometrics models with constant coefficients $b_{i}$ (5.5). Instead of Eq. (5.5) we have

$$
\rho_{i}(t)=a_{i}+\psi_{i}(t) M(t)+\epsilon_{i}(t)
$$

where we collect local differences in coefficients $b_{i}$ :

$$
\psi_{i}(t)=b_{i}+\left\{\begin{array}{l}
f(t)-a R_{i}, \text { if } f>a R_{i} \\
0, \text { else. }
\end{array}\right.
$$

In according to the initial model of adaptation, there is local difference between individuals in factor values, $f=f_{0}(t)+f_{i}(t)$, but such a model includes too many parameters, and we propose Eq. (5.7) as a compromise. Already this oversimplified one-factor approximation differs from the original model (5.5). Coefficient $\psi_{i}(t)$ depends on $t$, and increases in time of breakdowns together with its variance. This increase could explain difference between the simplest realization of econometrics models (5.5) and real time series, which was described in Ref. [25] (at least, it describes qualitatively local explosions of variability and of correlations between stocks in the crisis time).

\subsubsection{How to Merge Factors?}

Formally, for $q$ factors one can generalize the one-factor tension-driven Selye model (5.1) in the form.

$$
x_{k}=x_{k}\left(\psi_{1}, \psi_{2}, \ldots \psi_{q}\right)+\epsilon_{k} .
$$

In this equation, the compensated values of factors, $\psi_{i}=f_{i}-a_{i} r_{i}$, are used and $\sum_{i=1}^{q} r_{i} \leq R$.

Two questions appear immediately: (i) how to find the distribution of resource, assigned for neutralization of different factors, and (ii) how to represent the functions $x_{k}\left(\psi_{1}, \ldots \psi_{q}\right)$. Usually, in factor analysis and in physics both, we start from assumption of linearity ("in the first approximation"), but this approximation does not work here properly. In the simplest but reasonable approximation, max-min operations appear instead of linear operations. This sounds very modern [26] and even a bit extravagant, but it was discovered many years ago by Justus von Liebig (1840). His "law of the minimum" states that growth is controlled by the scarcest resource (limiting factor) [48]. This concept was originally applied to plant or crop growth. Many times it was criticized, rejected, and then returned and demonstrated quantitative agreement with experiments [48], [40], [5]. Liebig's Law of the minimum was extended to more a general conception of factors, not only for elementary physical description of available chemical substances and energy. Any environmental factor essential for life that is below the critical minimum, or that exceeds the maximum tolerable level could be considered as a limiting one.

The biological generalizations of Liebig's Law were supported by the biochemical idea of limiting reaction steps (modern theory of limiting steps and dominant systems for multiscale reaction networks is presented in recent review [20]). Some of generalizations went quite far from the agriculture and ecology. The law of the minimum was applied to economics [9] and to education, for example [39]. 
In according to Liebig's Law, the linear tension-driven model is

$$
x_{k}=\mu_{k}+l_{k} \max _{1 \leq i \leq q}\left\{\psi_{i}\right\}+\epsilon_{k} .
$$

The form of the response-driven model depends on the factor intensities:

$$
x_{k}=\mu_{k}+\sum_{i=1}^{q} b_{k i} a_{i} r_{i}+\epsilon_{k} .
$$

Analogously, the tension-and-response driven model reads:

$$
x_{k}=\mu_{k}+l_{k} \max _{1 \leq i \leq q}\left\{\psi_{i}\right\}+\sum_{i=1}^{q} b_{k i} a_{i} r_{i}+\epsilon_{k} .
$$

\subsubsection{Optimality and Fitness}

Adaptation optimizes the state of the system for a given amount of available resource. This idea seems very natural, but it may be a difficult task to find the objective function that is hidden behind the adaptation process. Nevertheless, even an assumption about existence of an objective functions and about its general properties helps in analysis of adaptation process.

Assume that adaptation should maximize an objective function $W$ which depends on the compensated values of factors, $\psi_{i}=f_{i}-a_{i} r_{i}$ for the given amount of available resource:

$$
\left\{\begin{array}{l}
W\left(f_{1}-a_{1} r_{1}, f_{2}-a_{2} r_{2}, \ldots f_{q}-a_{q} r_{q}\right) \rightarrow \max \\
r_{i} \geq 0, f_{i}-a_{i} r_{i} \geq 0, \sum_{i=1}^{q} r_{i} \leq R
\end{array}\right.
$$

The only question is: why can we be sure that adaptation follows any optimality principle? Existence of optimality is proven for microevolution processes and ecological succession. The mathematical backgrounds for the notion of "natural selection" in these situations are well-established after works of Haldane (1932) [22] and Gause (1934) [14]. Now this direction with various concepts of fitness (or "generalized fitness") optimization is elaborated in many details (see, for example, review papers $[2,37,18])$.

The foundation of optimization is not so clear for such processes as modifications of phenotype, and for adaptation in various time scales. The idea of genocopy-phenocopy interchangeability was formulated long ago by biologists to explain many experimental effects: the phenotype modifications simulate the optimal genotype ([60], p. 117). The idea of convergence of genetic and environmental effects was supported by analysis of genome regulation [61] (the principle of concentration-affinity equivalence). The phenotype modifications produce the the same change, as evolution of genotype does, but faster and in smaller range of conditions (the proper evolution can go further, but slower). It is natural to assume that adaptation in different time scales also follows the same direction, as evolution and phenotype modifications, but faster and for smaller changes. This hypothesis could be supported by many biological data and plausible reasoning. 


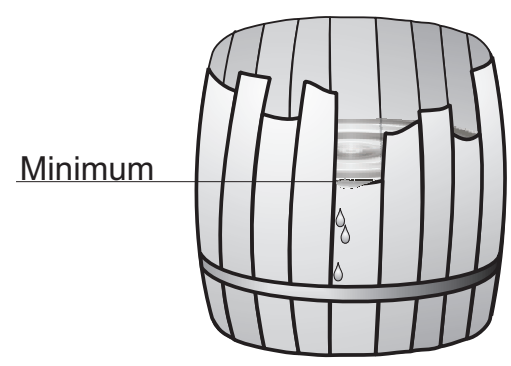

Figure 19: Liebig's barrel: the capacity of the barrel is limited by the shortest stave.

For man-made systems (social and economical ones) the idea of optimality is very natural if we keep in mind that "optimal" does not mean "perfect" and is always subject to the choice of objective functional and conditions.

It seems productive to accept the idea of optimality and to use it as far, as this will not contradict the data.

\subsection{Law of the Minimum Paradox}

Liebig used the image of a barrel - now called Liebig's barrel - to explain his law. Just as the capacity of a barrel with staves of unequal length is limited by the shortest stave, so a plant's growth is limited by the nutrient in shortest supply.

Adaptation system acts as a cooper and repairs the shortest stave to improve the barrel capacity.

Indeed, in well-adapted systems the limiting factor should be compensated as far as this is possible. It seems obvious because of very natural idea of optimality, but arguments of this type in biology should be considered with care.

Assume that adaptation should maximize a objective function $W(5.12)$ which satisfies Liebig's Law:

$$
W=W\left(\max _{1 \leq i \leq q}\left\{f_{i}-a_{i} r_{i}\right\}\right) ; \frac{\partial W(x)}{\partial x} \leq 0
$$

under conditions $r_{i} \geq 0, f_{i}-a_{i} r_{i} \geq 0, \sum_{i=1}^{q} r_{i} \leq R$. (Let us remind that $f_{i} \geq 0$ for all $i$.)

Description of the maximizers of $W$ gives the following theorem (the proof is a straightforward consequence of Liebig's Law and monotonicity of $W$ ).

Theorem 1. For any objective function $W$ that satisfies conditions (5.13) the optimizers $r_{i}$ are defined by the following algorithm.

1. Order intensities of factors: $f_{i_{1}} \geq f_{i_{1}} \geq \ldots f_{i_{q}}$.

2. Calculate differences $\Delta_{j}=f_{i_{j}}-f_{i_{j+1}}$ (take formally $\Delta_{0}=\Delta_{q+1}=0$ ).

3. Find such $k(0 \leq k \leq q)$ that

$$
\sum_{j=1}^{k}\left(\sum_{p=1}^{j} \frac{1}{a_{p}}\right) \Delta_{j} \leq R \leq \sum_{j=1}^{k+1}\left(\sum_{p=1}^{j} \frac{1}{a_{p}}\right) \Delta_{j}
$$




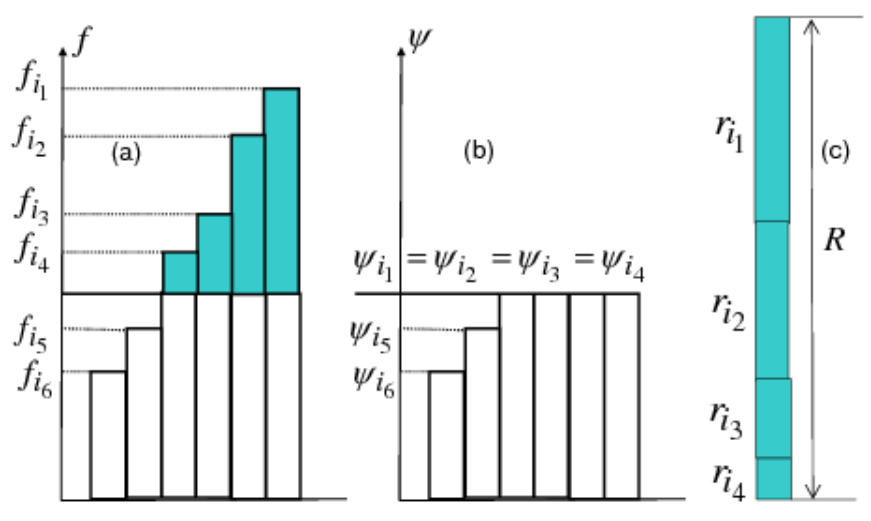

Figure 20: Optimal distribution of resource for neutralization of factors under Liebig's Law. (a) histogram of factors intensity (the compensated parts of factors are highlighted, $k=3$ ), (b) distribution of tensions $\psi_{i}$ after adaptation becomes more uniform, (c) the sum of distributed resources. For simplicity of the picture, we take here all $a_{i}=1$.

For $R<\Delta_{1}$ we put $k=0$, for $R>\sum_{j=1}^{k+1} j \Delta_{j}$ we take $k=q$.

4. If $k<q$ then the optimal amount of resource $r_{j_{l}}$ is

$$
r_{j_{l}}=\left\{\begin{array}{l}
\frac{\Delta_{l}}{a_{l}}+\frac{1}{a_{l} \sum_{p=1}^{k} \frac{1}{a_{p}}}\left(R-\sum_{j=1}^{k}\left(\sum_{p=1}^{j} \frac{1}{a_{p}}\right) \Delta_{j}\right), \text { if } l \leq k+1 ; \\
0, \quad \text { if } l>k+1 .
\end{array}\right.
$$

$$
\text { If } k=q \text { then } r_{i}=f_{i} \text { for all } i \text {. }
$$

This optimization is illustrated in Fig. 20.

Hence, if the system satisfies the law of the minimum then the adaptation process makes more uniform the tension produced by different factors $\psi_{i}=f_{i}-a r_{i}$ (Fig. 20). Thus adaptation decreases the effect from the limiting factor and hides manifestations of Liebig's Law.

Under the assumption of optimality (5.12) the law of the minimum paradox becomes a theorem: if Liebig's Law is true then microevolution, ecological succession, phenotype modifications and adaptation decrease the role of the limiting factors and bring the tension produced by different factors together.

The cooper starts to repair Liebig's barrel from the shortest stave and after reparation the staves are more uniform, than they were before. This cooper may be microevolution, ecological succession, phenotype modifications, or adaptation. For the ecological succession this effect (Liebig's Law leads to its violation by succession) was described in Ref. [54]. For adaptation (and in general settings too) it was demonstrated in Ref. [19].

The law of minimum together with the idea of optimality (even without an explicit form of the objective function) gives us answers to both question: (i) we know now the optimal distribution of resource (5.14), assigned for neutralization of different factors, and (ii) we can choose the function $x_{k}\left(\psi_{1}, \ldots \psi_{q}\right)$ from several model forms (5.9), (5.10), (5.11). 


\subsection{Law of the Minimum Inverse Paradox}

The simplest formal example of "anti-Liebig's" organization of interaction between factors gives us the following dependence of fitness from two factors: $W=-f_{1} f_{2}$ : each of factors is neutral in the absence of another factor, but together they are harmful. This is an example of synergy: the whole is greater than the sum of its parts. (For our selection of axes direction, "greater" means "more harm".) Let us give the formal definition of the synergistic system of factors for the given fitness function $W$.

Definition 2. The system of factors $F_{1}, \ldots F_{q}$ is synergistic, if for any two different vectors of their admissible values $\mathbf{f}=\left(f_{1}, \ldots f_{q}\right)$ and $\mathbf{g}=\left(g_{1}, \ldots g_{q}\right)$ ( $\left.\mathbf{f} \neq \mathbf{g}\right)$ the value of fitness at the average point $(\mathbf{f}+\mathbf{g}) / 2$ is less, than at the best of points $\mathbf{f}, \mathbf{g}$ :

$$
W\left(\frac{\mathbf{f}+\mathbf{g}}{2}\right)<\max \{W(\mathbf{f}), W(\mathbf{g})\} .
$$

Liebig's systems of factors violate the synergy inequality (5.15): if at points $\mathbf{f}$, $g$ with the same values of fitness $W(\mathbf{f})=W(\mathbf{g})$ different factors are limiting, then at the average point the value of both these factors are smaller, and the harm of the limiting factor at that point is less, than at both points $\mathbf{f}, \mathbf{g}$, i.e. the fitness at the average point is larger.

The fitness function $W$ for synergistic systems has a property that makes the solution of optimization problems much simpler. This proposition follows from the definition of convexity and standard facts about convex sets (see, for example, [47])

Proposition 3. The synergy inequality (5.15) holds if and only if all the sublevel sets $\{\mathbf{f} \mid W(\mathbf{f}) \leq$ $\alpha\}$ are strictly convex.

(The fitness itself may be a non-convex function.)

This proposition immediately implies that the synergy inequality is invariant with respect to increasing monotonic transformations of $W$. This invariance with respect to nonlinear change of scale is very important, because usually we don't know the values of function $W$.

Proposition 4. If the synergy inequality (5.15) holds for a function $W$, then it holds for a function $W_{\theta}=\theta(W)$, where $\theta(x)$ is an arbitrary strictly monotonic function of one variable.

Already this property allows us to study the problem about optimal distribution of the adaptation resource without further knowledge about the fitness function.

Assume that adaptation should maximize an objective function $W\left(f_{1}-r_{1}, \ldots f_{q}-r_{q}\right)(5.12)$ which satisfies the synergy inequality (5.15) under conditions $r_{i} \geq 0, f_{i}-a_{i} r_{i} \geq 0, \sum_{i=1}^{q} r_{i} \leq R$. (Let us remind that $f_{i} \geq 0$ for all $i$.) Following our previous convention about axes directions all factors are harmful and $W$ is monotonically decreasing function

$$
\frac{\partial W\left(f_{1}, \ldots f_{q}\right)}{\partial f_{i}}<0
$$


We need also a technical assumption that $W$ is defined on a convex set in $\mathbb{R}_{+}^{q}$ and if it is defined for a nonnegative point $\mathbf{f}$, then it is also defined at any nonnegative point $\mathbf{g} \leq \mathbf{f}$ (this inequality means that $g_{i} \leq f_{i}$ for all $\left.i=1, \ldots q\right)$.

The set of possible maximizers is finite. For every group of factors $F_{i_{1}}, \ldots F_{i_{j+1}},(1 \leq j+1<q)$ with the property

$$
\sum_{k=1}^{j} \frac{f_{i_{k}}}{a_{i_{k}}}<R \leq \sum_{k=1}^{j+1} \frac{f_{i_{k}}}{a_{i_{k}}}
$$

we find a distribution of resource $\mathbf{r}_{\left\{i_{1}, \ldots i_{j+1}\right\}}=\left(r_{i_{1}}, \ldots r_{i_{j+1}}\right)$ :

$$
r_{i_{k}}=\frac{f_{i_{k}}}{a_{i_{k}}}(k=1, \ldots j), r_{i_{j+1}}=R-\sum_{k=1}^{j} \frac{f_{i_{k}}}{a_{i_{k}}}, \quad r_{i}=0 \text { for } i \notin\left\{i_{1}, \ldots i_{j+1}\right\}
$$

For $j=0$, Eq. (5.16) gives $0<R \leq f_{i_{1}}$ and there exists only one nonzero component in the distribution (5.17), $r_{i_{1}}=R / a_{i_{1}}$.

We get the following theorem as an application of standard results about extreme points of convex sets [47].

Theorem 5. Any maximizer for $W\left(f_{1}-r_{1}, \ldots f_{q}-r_{q}\right)$ under given conditions has the form $\mathbf{r}_{\left\{i_{1}, \ldots i_{j+1}\right\}}$ (5.17).

If the initial distribution of factors intensities, $\mathbf{f}=\left(f_{1}, \ldots f_{q}\right)$, is almost uniform and all factors are significant then, after adaptation, the distribution of effective tensions, $\psi=\left(\psi_{1}, \ldots \psi_{q}\right)$ $\left(\psi_{i}=f_{i}-a_{i} r_{i}\right)$, is less uniform. Following Theorem 2, some of factors may be completely neutralized and one additional factor may be neutralized partially. This situation is opposite to adaptation to Liebig's system of factors, where amount of significant factors increases and the distribution of tensions becomes more uniform because of adaptation. For Liebig's system, adaptation transforms low dimensional picture (one limiting factor) into high dimensional one, and we expect the well-adapted systems have less correlations than in stress. For synergistic systems, adaptation transforms high dimensional picture into low dimensional one (less factors), and our expectations are inverse: we expect the well-adapted systems have more correlations than in stress (this situation is illustrated in Fig. 21; compare to Fig. 20). We call this property of adaptation to synergistic system of factors the law of the minimum inverse paradox.

The fitness by itself is a theoretical construction based on the average reproduction coefficient (instant fitness). It is impossible to measure this quantity in time intervals that are much shorter than the life length. Hence, to understand which system of factors we deal with, Liebig's or synergistic one, we have to compare theoretical consequences of their properties. First of all, we can measure results of adaptation, and use for analysis properties of optimal adaptation in ensembles of systems for analysis (Fig. 20, Fig. 21).

There are some evidences about existence of synergistic systems of factors. For example, the aftersurgical rehabilitation of people suffering the lung cancer of the III and IV clinical groups was studied [28]. Dynamics of variance and correlations for them have directions which are unusual 

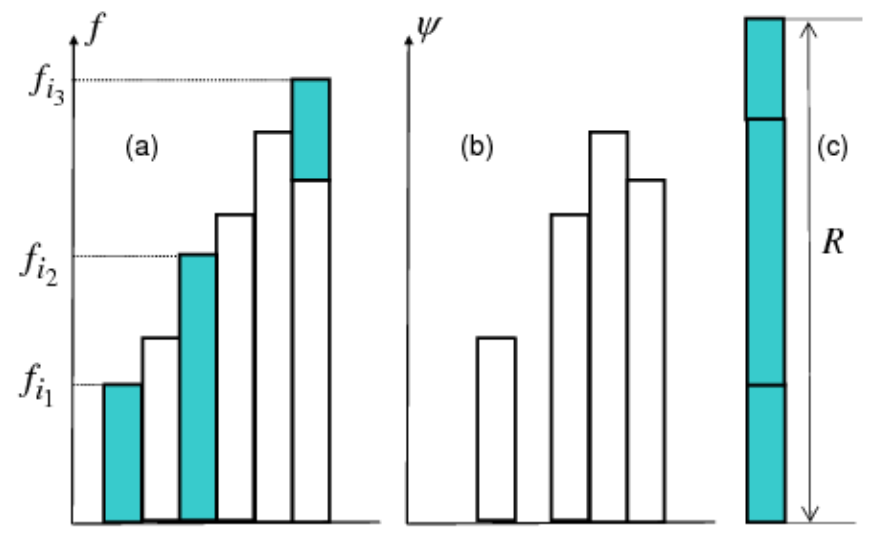

Figure 21: Typical optimal distribution of resource for neutralization of synergistic factors. (a) Factors intensity (the compensated parts of factors are highlighted, $j=2$ ), (b) distribution of tensions $\psi_{i}$ after adaptation becomes less uniform (compare to Fig. 20), (c) the sum of distributed resources. For simplicity of the picture, we take here all $a_{i}=1$.

for Liebig's systems: increase of the correlation corresponds to decrease of variance. Moreover, analysis of maxima and minima of correlations and mortality demonstrates that in this case increase of correlations corresponds to decrease of stress. Hence, in Ref. [28] the hypothesis was suggested that in this case some factors superlinear increase harmfulness of other factors, and this is the example of a synergistic system of factors.

\subsection{Adaptation Dynamics Near a Border of Existence}

We used the idea of adaptation resource to demonstrate how can the models of adaptation explain the observable dynamics of correlation and variance under environmental loads. Another theoretical approach to explain these effects was proposed in Ref. [46]. The idea is quite simple and attractive. Let us represent the population of systems by points in a bounded set $U \subset \mathbb{R}^{q}$ with smooth boundary. Coordinates correspond to the internal characteristics of the systems. The whole population is described by a continuous density function $u(x)$ in $U$.

The hypothetical behaviour of the individual system can be described as a random walk in $U$, and the density dynamics satisfies the correspondent Fokker-Planck equation

$$
\partial_{t} u=-(\nabla, b u)+a \triangle u
$$

where $a>0$ is a constant diffusion coefficient and $b=b(x)$ is a drift vector. After analysis of various optimality conditions, the asymptotic behaviour of solution of Eq. (5.18) was studied for $b=A b_{0}, b_{0}=$ const $\left(\left\|b_{0}\right\|=1\right), A \rightarrow \infty$, and for no-flux boundary conditions $(b u-a \nabla u, \nu)_{\partial U}=$ 0 (where $\nu$ is the outer normal to $\partial U$ ). The drift vector $b$ models the pressure of environmental factors. For large $A$, the drift presses the distribution to the boundary of $U$, dimension decreases, 
and correlations increase. The asymptotics for large $A$ is determined by a tangent paraboloid to $\partial U$ at the point $x_{b}$, where the linear function $(b, x)$ achieves the maximal value on $\partial U$ (generically, such a point us unique). At this point $\nu=b_{0}$. The asymptotic of the solution coincides in the main term with exact analytical solution to the Fokker-Planck equation (5.18) with constant coefficients in a paraboloid.

Let us select a rectangular coordinate system with origin at the point $x_{b}$, such that in this system $b=(0, \ldots, 0,-A)$ and $U$ is given by the inequality $x_{q} \geq \sum_{i=1}^{q-1} \lambda_{i} x_{i}^{2}+o\left(\sum_{i=1}^{q-1} x_{i}^{2}\right)$. In this coordinate system, the solution to the problem is $u(x)=v \exp \left(-\frac{A x_{q}}{a}\right)$ with constant $v$. For large $A$, the variance of $x_{q}, \sigma_{q}^{2} \sim \frac{a^{2}}{A^{2}}$, the marginal distribution of $x_{1}, \ldots x_{q-1}$ is almost Gaussian with $\sigma_{i}^{2}=\frac{a}{A \lambda_{i}}$, and these variables are almost independent. We can see that the concentration ellipsoid for $x_{1}, \ldots x_{q-1}$ does not change its form with increase of $A$ : the ratio $\frac{\sigma_{i}^{2}}{\sigma_{j}^{2}}=\frac{\lambda_{j}}{\lambda_{i}}$ is independent of $A$. On the other hand, contraction in direction $x_{q}$ with increase of $A$ goes faster, and $\frac{\sigma_{q}^{2}}{\sigma_{i}^{2}} \rightarrow 0$ as $\frac{a \lambda_{i}}{A}$ $(i=1, \ldots q-1)$.

To check which mechanism is closer to reality we have to resolve the "disk-or-bullet" alternative: How does the correlation increase: (i) the largest eigenvalues of the correlation matrix increase and the ellipsoid of concentration becomes stretched-out in small amount of dimensions (presumably, in one, that is the bullet form) or (ii) the smallest eigenvalue goes fast to zero, and the ellipsoid of concentration becomes flattened out (the disk or the pancake form). The existing data ensure us that this alternative should be resolved in favor of bullet: under the pressure of environment factors, the small amount of largest eigenvalues increases. It is necessary to mention that the variance decreases in the Fokker-Planck model near the border of existence, that also contradicts the majority of existing observation.

\subsection{Dynamical Models of Adaptation}

\subsubsection{Qualitative Assumptions}

In this subsection, we describe the problem of dynamical modeling of adaptation. We have not a complete theory now, but rather a combination of qualitative assumptions and attempts of their formalization.

The factor-resource models of adaptation presented in paper describe single distribution of the adaptation resource: they give the optimal distribution of the adaptation resource, if a system has some amount of resource available and experiences given loads of environmental factors. But the situation changes in time, and a dynamical theory of resource distribution is necessary.

Since Selye's work [52] researchers try to summarize the main qualitative assumptions about the hypothetical "adaptation energy" (AE). The list of assumptions based on Selye's works and years of application of this notion is published in Ref. [49]:

1. AE is a finite supply, presented at birth (Selye).

2. As a protective mechanism, there is some upper limit to the amount of $\mathrm{AE}$ that an individual can use at any discrete moment in time. It can be focused on one activity, or divided among 
other activities designed to respond to multiply occupational challenges.

3. There is a threshold of AE activation that must be present to potentiate an occupational response.

4. AE is active at two levels of awareness: a primary level at which creating the response occurs at a high awareness level, with high usage of finite supply of adaptation energy; and a secondary level at which the response creation is being processing at a sub-awareness level, with a lower energy expenditure.

The first assumption appears to be incompatible with clinical observations [16]. Instead of them, B. Goldstone proposed an assumption that is more plausible "from the general practitioner point of view":

- (1GP) Adaptation Energy can be created, though the income of this energy is slower in old age; it can also be stored as Adaptation Capital, though the storage capacity has a fixed limit. If an individual spends his Adaptation Energy faster than he creates it, he will have to draw on his capital reserve; when this is exhausted he dies.

In addition, B. Goldstone formulated several practical observations about interaction of stress stimulus which are typical, but apparently contradictory. Each of these answers is probably true in different circumstances. The proper theory of adaptation has to demonstrate all these types of qualitative behavior.

1. If an individual is failing to adapt to a disease he may succeed in so doing, if he is exposed to a totally different mild stimulus (such as slight fall of oxygen tension).

2. In the process of adapting to this new stimulus he may acquire the power of reacting more intensely to all stimuli.

3. As a result of a severe stimulus an individual may not be able to adapt successfully to a second severe stimulus (such as a disease).

4. If he is already adapting successfully to a disease this adaptation may fail when he is exposed to a second severe stimulus.

5. In some diseases (those of Adaptation) exposure to a fresh severe stimulus may cure the disease. Here, too, exposure to an additional stressor will bring him nearer to death but the risk may be justifiable if it is likely to re-mould the adaptive mechanism to a normal form.

The analogy with financial economics was also mentioned: "These apparently paradoxical results may be explained on the same simple lines as the individual's own financial balance sheet. Whether he spends his money in one way or another, he is subject to the same simple inexorable laws which relate income, expenditure and capital reserve with final bankruptcy" [16]. 


\subsubsection{The Model Structure}

Resource and Reserve. To cover non-physical and non-biological applications we will keep the term "adaptation resource" (AR) instead of "adaptation energy" (AE). We postulate that there are two "warehouses" for storage of AR with different availability or two forms of existence of this resource, namely, resource and reserve. In the resource form, AR is available immediately for all current needs (with limited rate of supply). The resource form could be produced by the organism (with limited rate of production), or the consumption of the resource can be compensated from the reserve: after some threshold of demand the reserve opens, and closes again when the closing threshold is achieved. Recourse can be produced by the organism (with limited rate).

Resources Degradation. Amount of resource $r_{i}$ assigned for neutralization of an environmental factor $f_{i}$ slowly decreases by the linear kinetic law:

$$
\frac{\mathrm{d} r_{i}}{\mathrm{~d} t}=-k_{\mathrm{d}} r_{i}+v_{i}
$$

where $k_{\mathrm{d}}$ is the degradation rate constant, $v_{i}$ is the intensity of the external supply of AR for neutralization of the environmental factor $f_{i}$.

Resources Conversion. In the normal state (with closed reserve) it is impossible to reassign AR from neutralization of one factor $f_{i}$ towards neutralization of another factor $f_{j}(i \neq j)$. But together with opening of the reserve, a possibility of reassignment (conversion) appears: with some loss of $\mathrm{AR}$ and (the exchange margin), $r_{i}$ could be transformed into $r_{j}$. The exchange rate is limited. Therefore, for systems with resource conversion instead of degradation equation (5.19) we have to write:

$$
\frac{\mathrm{d} r_{i}}{\mathrm{~d} t}=-k_{\mathrm{d}} r_{i}+\nu \sum_{j, j \neq i} w_{i j}-\sum_{j, j \neq i} w_{j i}+v_{i},
$$

where $0<\nu<1$ is the conversion coefficient ( $1-\nu$ is the conversion margin), $w_{i j} \geq 0$ is the rate of transformation of $r_{j}$ into $r_{i}, v_{i}$ is the intensity of the external supply of AR from the resource warehouse for neutralization of the environmental factor $f_{i}$.

Fitness and Generalized Affinity. For kinetic equations, there are two typical problems: (i) how to derive the rate functions and (ii) where to take the kinetic constants (if the general form of the rate function is found). The clue idea is: the flux of AR has to increase fitness: for transition $r_{i} \rightarrow \nu r_{j}\left(r_{i}>0\right)$ (conversion of $r_{i}$ into $r_{j}$ with conversion coefficient the rate $w_{j i}$ ) should depend on $\nu \partial W / \partial r_{j}-\partial W / \partial r_{i}$ :

$$
w_{j i}=\left\{\begin{array}{l}
0, \text { if } \nu \frac{\partial W}{\partial r_{j}}-\frac{\partial W}{\partial r_{i}} \leq 0 ; \\
\omega_{j i}\left(\nu \frac{\partial W}{\partial r_{j}}-\frac{\partial W}{\partial r_{i}}\right), \text { if } \nu \frac{\partial W}{\partial r_{j}}-\frac{\partial W}{\partial r_{i}}>0 \text { and } r_{i}>0,
\end{array}\right.
$$

where $\omega_{j i}$ is a non-negative quantity. 
Due to analogy with chemical kinetics and thermodynamics [10,13], we can call the nonnegative quantity

$$
A_{j i}=\left\{\begin{array}{l}
0, \text { if } \nu \frac{\partial W}{\partial r_{j}}-\frac{\partial W}{\partial r_{i}} \leq 0 \\
\nu \frac{\partial W}{\partial r_{j}}-\frac{\partial W}{\partial r_{i}}, \text { if } \nu \frac{\partial W}{\partial r_{j}}-\frac{\partial W}{\partial r_{i}}>0,
\end{array}\right.
$$

the generalized affinity. The rate of the fitness gain in the conversion process $r_{i} \rightarrow \nu r_{j}\left(r_{i}>0\right)$ is $\omega_{j i} A_{j i} \geq 0$. The generalized affinity $A_{j i}$ characterizes how effectively the conversion process increases the fitness.

Basically, there are two simple reasonable choices of $\omega_{j i}$ :

- $\omega_{j i}=k_{j i} h\left(r_{i}\right)$, where $h(x)$ is the Heaviside step function;

- $\omega_{j i}=k_{j i} r_{i}$

The conversion kinetic constant $k_{i j}$ depends on indices $i, j$, but in the simplest models we can evaluate it by a single constant $k$. The first choice, $\omega_{j i}=$ const, assumes that the rate of conversion process is determined by the demand measured by the generalized affinity. If the dynamics follows this choice then the resource amount $r_{i}$ can be exhausted in finite time. Due to the second choice, $\omega_{j i}=$ const $r_{i}$, the variable $r_{i}$ will never achieve zero from the positive initial conditions $r_{i}>0$. The choice between these possibilities is not obvious.

We have to stress that the conversion process appear in a state of open reserve.

Dynamics of Available Free AR. For amount of available free resource we use notation $r_{0}$. The limits of $r_{0}$ values are fixed: $0 \leq r_{0} \leq R_{0}$. (Perhaps, $R_{0}$ could slowly change in time, to decrease because of ageing or to increase because of training, but we postpone the discussion of this possibility.)

Similarly to (5.20) we can write

$$
\frac{\mathrm{d} r_{0}}{\mathrm{~d} t}=-k_{\mathrm{d}} r_{0}-\sum_{j} v_{j}+v_{0},
$$

where $v_{j}$ is the rate of assignment $r_{0} \rightarrow r_{j}\left(r_{0}>0\right)$, and $v_{0}$ is the rate of $r_{0}$ expense recovering. For description of rates $v_{j}$, let us introduce the generalized affinities $A_{j}$ of processes $r_{0} \rightarrow r_{j}\left(r_{0}>0\right)$ : $A_{j}=\partial W / \partial r_{j} \geq 0$. With these affinities we can write:

$$
v_{i}=\omega_{i} A_{i}
$$

where $\omega_{i}$ is a non-negative quantity. For $\omega_{i}$ we have two simple possibilities, again: either $\omega_{i}=$ const $h\left(r_{0}\right)$ or $\omega_{i}=$ const $r_{0}$. Here $h(x)$ is the Heaviside unite step function. The first choice can lead to the full exhaustion of the available resource in finite time, the second choice guarantees positivity: $r_{0}(t)>0$ for $t>0$ if $r_{0}(0)>0$.

The recovering term $v_{0}$ consists of two parts. First of all, this is the normal production $v_{0}^{\mathrm{n}}$. It depends on the deficit $R_{0}-r_{0}$ and on the general wellbeing that can be measured by the fitness $W$. For example, if we assume that $W$ is positive, then $v_{0}^{\mathrm{n}}$ may be just a product const $\left(R_{0}-r_{0}\right) W$, 
or a function with saturation: $v_{0}^{\mathrm{n}}=a\left(R_{0}-r_{0}\right) W /\left(1+b\left(R_{0}-r_{0}\right) W\right)$ with positive constants $a, b$. Secondly, if the reserve is open, then there is the flow from the reserve to the available free resource, $v_{0}^{\mathrm{r}}$. The simplest hypothesis is that $v_{0}^{\mathrm{r}}$ is either constant (if the reserve storage is not empty) or proportional to the amount of AR in the reserve storage.

Opening and Closing of AR reserve. There may exist many signals for opening and closing of the AR reserve. One of them is the level of the available AR resource $r_{0}$. If the reserve is closed and this level becomes too low (achieves the lower threshold value, $\underline{r}$ ), then the reserve opens and a flux from the reserve to the available resource appears. If the reserve is open, and the level of the available AR resource achieves the upper threshold value $\bar{r}>\underline{r}$, then the reserve closes. Hysteresis in reserve opening/closure can cause some of effects mentioned by B. Goldstone [16]. This hysteresis implies that strong adaptive answer to environmental factors load starts after exhausting of freely available AR. This peculiarity of dynamics can be interpreted as tolerance to small loads and meets Shelford's Law of Tolerance ([36], Chapter 5) (partially at least).

Dynamics of Reserve AR. Similarly to dynamics of the available free AR, the amount of reserve $\mathrm{AR}, r_{\mathrm{rv}}$, has the upper limit, $0 \leq r_{\mathrm{rv}} \leq R_{\mathrm{rv}}$. The upper boundary $R_{\mathrm{rv}}$ changes slowly with ageing and wellbeing changes.

$$
\frac{\mathrm{d} r_{\mathrm{rv}}}{\mathrm{d} t}=-k_{\mathrm{d}} r_{\mathrm{rv}}-v_{0}^{\mathrm{r}}+v_{\text {prod }},
$$

where $v_{0}^{\mathrm{r}}$ is the flux from the reserve $\mathrm{AR}$ to the available free $\mathrm{AR}$, and $v_{\text {prod }}$ is the rate of the reserve AR production. The production term $v_{\text {prod }}$ depends on the deficit $R_{\mathrm{rv}}-r_{\mathrm{rv}}$ and on the general wellbeing that can be measured by the fitness $W$.

Simple Model for Adaptation to Single Factor. Variables: $r$ - amount of AR assigned for neutralization of an external factor $F ; r_{0}$ - amount of available free AR, $r_{\mathrm{rv}}$ - amount of reserve $\mathrm{AR}$, and a boolean variable $B_{\mathrm{o} / \mathrm{c}}-$ "open/closed" reserve $\left(B_{\mathrm{o} / \mathrm{c}}=1\right.$ if reserve is open, and $B_{\mathrm{o} / \mathrm{c}}=$ 0 if it is closed). Parameters: capacities $R_{0}$ and $R_{\mathrm{rv}}$, constant of degradation $k_{\mathrm{d}}$, efficiency of resource for neutralization of factor $-a$, lower threshold for reserve opening, $\underline{r}$, upper threshold for reserve closing, $\bar{r}$, and kinetic constants: for degradation $k_{\mathrm{d}}$, for resource assignment $k$, for the reserve-available resource transformation $k_{\mathrm{rv}}$ and for recovering (production) $k_{\mathrm{pr}}$.

Intensity of the factor is $f$. Compensated values of the factor is $\psi=f-a r$. For dynamical models $f$ depends on time and the overcompensation may occur with $f<a r$. The overcompensation is not better than simple compensation, and the fitness $W$ is function of $\psi h(\psi)$ : $W=\left(W_{0}-\psi^{2} h(\psi)\right) h\left(W_{0}-\psi^{2} h(\psi)\right)$. Zero fitness means death. Simple scaling allows us to take $a=1$. 
The simplest dynamical model has three real variables and one Boolean:

$$
\begin{aligned}
\frac{\mathrm{d} r}{\mathrm{~d} t}= & -k_{\mathrm{d}} r+k r_{0}(f-r) h(f-r) ; \\
\frac{\mathrm{d} r_{0}}{\mathrm{~d} t}= & -k_{\mathrm{d}} r_{0}-k r_{0}(f-r) h(f-r)+k_{\mathrm{rv}} B_{\mathrm{o} / \mathrm{c}} r_{\mathrm{rv}}\left(R_{0}-r_{0}\right) \\
& +k_{\mathrm{pr}}\left(R_{0}-r_{0}\right) W \\
\frac{\mathrm{d} r_{\mathrm{rv}}}{\mathrm{d} t}= & -k_{\mathrm{d}} r_{\mathrm{rv}}-k_{\mathrm{rv}} B_{\mathrm{o} / \mathrm{c}} r_{\mathrm{rv}}\left(R_{0}-r_{0}\right)+k_{\mathrm{pr}}\left(R_{\mathrm{rv}}-r_{\mathrm{rv}}\right) W
\end{aligned}
$$

If reserve is open then $r_{0}<\bar{r}$. It closes when $r_{0}=\bar{r}$. If reserve is closed then $r_{0}>\underline{r}$. It opens when $r_{0}=\underline{r}$. This hysteresis can cause oscillations in adaptation for some interval of the factor load.

Detailed qualitative study of system (5.26) and its generalizations together with the problem of optimal control of such systems is a part of the future research program.

\section{Discussion}

\subsection{What do we know now for sure?}

We study a universal effect in ensembles of similar systems under load of similar factors: in crisis, typically, correlation increases, and, at the same time, variance (and volatility) increases too. After the crisis achieves its bottom, it can develop into two directions: recovering (both correlations and variance decrease) or fatal catastrophe (correlations decrease, but variance not). Three results are presented in the paper:

- Existence of the effect is demonstrated by analysis of many experiments and observation of groups of humans, mice, trees, grassy plants, and financial time series;

- We propose an explanation of the effect through general properties of ensembles of similar systems which are adapting to the same or similar environment. A hierarchy of models of adaptation is developed;

- We analyze various types of interaction between environmental factors and prove that different organization of this interaction (Liebig's versus synergistic systems) leads to different adaptation dynamics.

In econophysics, as well as in biophysics, a similar phenomenon is described: under pressure of environmental factors the correlation between systems, as well as between attributes, increases and the variance between systems (variability), as well as the variance in time (volatility), also increases.

This typical behavior can be changed when the tension becomes too strong. In that case, correlation and variance change in antiphase: when the situation gets worse correlation decreases 
(variance increases), and in recovery the inverse, correlation increases (variance decreases). This effect was observed several times, but perhaps needs additional confirmation.

The case study of the thirty largest companies from British stock market for the period 20062008 supports the main hypothesis. It is also demonstrated that the correlation in time (between daily data) also has diagnostic power (as well as the correlation between companies has) and connections between days (Figs. 16, 18) may clearly indicate and, sometimes, predict the chronology of the crisis.

The principal component analysis demonstrates that the largest eigenvalues of the correlation matrices increase in crisis and under environmental pressure (before the inverse effect "on the other side of crisis" appears). Different methods for selection of significant principal components, Kaiser's rule, random matrix approach and the broken stick model, give similar results in a case study. Kaiser's rule gives more principal components than two other methods and the higher sensitivity of the indicator $\operatorname{Dim}_{\mathrm{K}}$ causes some difficulties in interpretation. The random matrix estimates select too small amount of components, and the indicator $\operatorname{Dim}_{M} P$ seems not sensitive enough. In our case study the best balance between sensitivity and stability gives the dimension, estimated by the broken stick model Dim $\mathrm{BS}$.

This effect is demonstrated for humans, mice, trees, grassy plants, and financial time series. The universality and broadness of confirmations ensure us that for its explanation should be found a theoretical idea of the adequate level of generality.

We propose to look for such an idea in the models of adaptation. The main hypothesis now is: the observed effects naturally appear in ensembles of similar systems adapting to similar environmental factors.

We have to stress that we don't consider here interaction between systems. For many examples there was no interaction between systems at all, neither one-to-one interaction nor mean-field type interaction through the common environment. Sometimes the interactions exists and may be important for amplification or diminishing the effect intensity, but following the empirical data we have to propose the theory which works mainly with individual difference and can take interaction into account as correction terms. In that sense, we do not consider markets as complex adaptive systems [31], but rather as ensembles of adaptive individuals.

Already a system of simple models of adaptation to one factor gives qualitative explanation of the effect.

For interaction of several factors two basic types of organization are considered: Liebig's systems and synergistic systems of factors. The adaptation process (as well as phenomodification, ecological succession, or microevolution) acts differently onto these systems of factors and makes Liebig's systems more uniform (instead of systems with limiting factor) and synergistic systems less uniform. These theorems give us two paradoxes which explain differences observed between artificial (less adapted) systems and natural (well adapted) systems.

Empirically, we expect the appearance of synergistic systems in extremely difficult conditions, when factors appear that superlinearly amplify the harm from other factors. Some data from oncological clinics already support these expectations. 


\subsection{Choice of Coordinates and the Problem of Invariance}

All indicators of the level of correlations are non-invariant with respect to transformations of coordinates. For example, rotation to the principal axis annuls all the correlations. Dynamics of variance also depends on nonlinear transformations of scales. Dimensionless variance of logarithms (or "relative variance") often demonstrates more stable behaviour especially when changes of mean values are large.

The observed effect depends on the choice of attributes. Nevertheless, many researchers observed it without a special choice of coordinate system. What does it mean? We can propose a hypothesis: the effect may be so strong that it is almost improbable to select a coordinate system where it vanishes. For example, if one accepts the one-factor tension-driven Selye model (5.1), (5.2) then observability of the effect means that for typical nonzero values of $\psi$ in crisis

$$
l_{k}^{2} \psi^{2}>\operatorname{var}\left(\epsilon_{k}\right)
$$

for more than one value of $k$, where var stands for variance of the noise component (this is sufficient for increase of the correlations). If

$$
\psi^{2} \sum_{k} l_{k}^{2} \gg \sum_{k} \operatorname{var}\left(\epsilon_{k}\right)
$$

and the set of allowable transformations of coordinates is bounded (together with the set of inverse transformations), then the probability to select randomly a coordinate system which violates condition (6.1) is small (for reasonable definitions of this probability and of the relation $\gg$ ).

On another hand, the choice of attributes is never random, and one can look for the reason of so wide observability of the effect in our (human) ways to construct the attribute systems.

\subsection{Problems and Programs}

There may be two programs for development of further research. First of all, the simplest system of models of adaptation should be fitted to various data, both economical and biophysical. These models could be easily incorporated in the toolboxes of data analysis. The classical econometrics [23] already deals with hidden factors, now we have just to use a special nonlinear model of adaptation to these factors.

Another possible direction is development of the dynamical models of adaptation. The optimization models of adaptation (5.12) describe a single action, distribution of adaptation resource. Any kinetic model introduces more parameters than one can find from experiment. Nevertheless, adaptation is a process in time. We have to create a system of models with minimal number of parameters. Delay in time is necessary, also we have to introduce several levels of resource availability with distinguishing of resource and reserve. The processes of resource recovery should be described.

In this paper, we discuss principles of kinetic models of adaptation and formulate a simple onefactor model (5.26).Detailed qualitative study of this system for various combinations of parameter values together with the problem of optimal control of this system is a part of the future research 
program. Many modifications and improvements of the model are obvious. For example, the degradation constant for reserve may be smaller than for other forms of AR, production of AR should perhaps be a function with saturation. It is necessary to include more realistic dose response curves (instead of linear one) including possibility of non-monotonic dependence, because low doses may actually cause greater impact than high doses for a specific response [7].

Of course, it is also necessary to develop and to study models with many factors. But any improvement introduces new unknown constants and necessity either to find these constants, or to produce the parametric portrait in space with additional dimensions. Now the first task is to reproduce the known qualitative effects in dynamics of adaptation and to develop an optimal control strategy.

Finally, the very intriguing question arises: how a phenomenon (or epiphenomenon) of a single resource appears, and what are the conditions of its existence and of its dynamical disintegration into various particular resources?

\section{Acknowledgements}

We are very grateful to many people for 21 year of collaboration, to our first co-author [19] V.T. Manchuk, to A.G. Abanov, G.F. Bulygin, R.A. Belousova, R.G. Khlebopros, G.B. Kofman, A.S. Mansurov, T.P. Mansurova, L.S. Mikitin, A.V. Pershin, L.I. Pokidysheva, M.G Polonskaya, L.D. Ponomarenko, V.N. Razzhevaikin, K.R. Sedov, S.M. Semenov, E.N. Shalamova, S.Y. Skobeleva, G.N. Svetlichnaia. Many physiological data were collected in Institute for Medical Problems of Northern Regions ${ }^{\ddagger}$.

\section{References}

[1] S. Breznitz (Ed.). The denial of stress. New York: International Universities Press, Inc., 1983.

[2] I.M. Bomze. Regularity vs. degeneracy in dynamics, games, and optimization: a unified approach to different aspects. SIAM Review, 44 (2002), 394-414.

[3] G.V. Bulygin, A.S. Mansurov, T.P. Mansurova, A.A. Mashanov, E.V. Smirnova. Impact of health on the ecological stress dynamics. Institute of Biophysics, Russian Academy of Sciences, Preprint 185B, Krasnoyarsk, 1992.

[4] G.V. Bulygin, A.S. Mansurov, T.P. Mansurova, E.V. Smirnova. Dynamics of parameters of human metabolic system during the short-term adaptation. Institute of Biophysics, Russian Academy of Sciences, Preprint 180B, Krasnoyarsk, 1992.

[5] B.S. Cade, J.W. Terrell, R.L. Schroeder. Estimating effects of limiting factors with regression quantiles. Ecology, 80 (1999), 311-323.

\footnotetext{
${ }^{\ddagger}$ State Research Institute for Medical Problems of Northern Regions, Siberian Branch of Russian (USSR) Academy of Medical Sciences (Krasnoyarsk).
} 
[6] R. Cangelosi, A. Goriely. Component retention in principal component analysis with application to cDNA microarray data. Biology Direct, 2 (2007). Online: http://www.biologydirect.com/content/2/1/2

[7] T. Colborn, D. Dumanoski, J.P. Meyers. Our stolen future: are we threatening our fertility, intelligence, and survival? - A Scientific Detective Story. Dutton, Peguin Books, NY, 1996.

[8] S. Çukur, M. Eryig̃it, R. Eryig̃t. Cross correlations in an emerging market financial data. Physica A, 376 (2007), 555-564.

[9] H.E. Daly. Population and economics - a bioeconomic analysis. Population and Environment, 12 (1991), 257-263.

[10] T. De Donder, P. Van Rysselberghe. Thermodynamic theory of affinity. A book of principles. Stanford: University Press, 1936.

[11] S. Drożdż, F. Grümmer, A.Z. Górski, F. Ruf, J. Speth. Dynamics of competition between collectivity and noise in the stock market. Physica A, 287 (2000) 440-449.

[12] A.C. Eliasson, C. Kreuter. On currency crisis: a continuous crisis definition (Deutsche Bank Research Quantitative Analysis Report), Conference paper, X International "Tor Vergata" Conference on Banking and Finance, December 2001.

[13] M. Feinberg. Chemical kinetics of a sertain class. Arch. Rat. Mech. Anal., 46 (1972), 1-41.

[14] G.F. Gause. The struggle for existence. Williams and Wilkins, Baltimore, 1934. Online: http://www.ggause.com/Contgau.htm.

[15] W.N. Goetzmann, L. Li, K.G. Rouwenhorst. Long-term global market correlations (October 7, 2004). Yale ICF Working Paper No. 08-04. Available at SSRN: http://ssrn.com/abstract=288421

[16] B. Goldstone. The general practitioner and the general adaptation syndrome. S. Afr. Med. J. 26 (1952), 88-92, 106-109. PMID: 14901129, 14913266.

[17] P. Gopikrishnan, B. Rosenow, L.A.N. Amaral, H.E. Stanley. Universal and Nonuniversal Properties of cross correlations in financial time series. Phys. Rev. Lett., 83 (1999), 14711474.

[18] A.N. Gorban. Selection theorem for systems with inheritance. Math. Model. Nat. Phenom., 2, No. 4, 2007, 1-45.

[19] A.N. Gorban, V.T. Manchuk, E.V. Petushkova (Smirnova). Dynamics of physiological parameters correlations and the ecological-evolutionary principle of polyfactoriality. Problemy Ekologicheskogo Monitoringa i Modelirovaniya Ekosistem [The Problems of Ecological Monitoring and Ecosystem Modelling], Vol. 10. Gidrometeoizdat, Leningrad, 1987, pp. 187-198. 
[20] A.N. Gorban, O. Radulescu. Dynamic and static limitation in multiscale reaction networks, Revisited. Adv. Chem. Eng., 34 (2008), 103-173.

[21] A.N. Gorban, E.V. Smirnova, T.A. Tyukina. Correlations, risk and crisis: from physiology to finance. Physica A, submitted. arXiv preprint: http://arxiv.org/abs/0905.0129 (May 1, 2009).

[22] J.B.S. Haldane. The causes of evolution. Princeton Science Library, Princeton University Press, 1990.

[23] G.G. Judge, W.E. Griffiths, R.C. Hill, H. Lütkepohl, T.-C. Lee. The theory and practice of econometrics. Wiley Series in Probability and Statistics, \# 49 (2nd ed.), Wiley, New York 1985.

[24] I.V. Karmanova, V.N. Razzhevaikin, M.I. Shpitonkov. Application of correlation adaptometry for estimating a response of herbaceous species to stress loadings. Doklady Botanical Sciences, 346-348 (1996), 4-7. [Translated from Doklady Akademii Nauk SSSR, 346, 1996.]

[25] F. Lillo, R.N. Mantegna. Variety and volatility in financial markets. Phys. Rev. E, 62 (2000), $6126(2000)$.

[26] G. L. Litvinov, V. P. Maslov (Eds.). Idempotent mathematics and mathematical physics. Contemporary Mathematics, AMS, Providence, RI, 2005.

[27] F. Longin, B. Solnik. Is the correlation in international equity returns constant: 1960-1990? J. Internat. Money and Finance, 14, No. 1 (1995), 3-26.

[28] A.S. Mansurov, T.P. Mansurova, E.V. Smirnova, L.S. Mikitin, A.V. Pershin. How do correlations between physiological parameters depend on the influence of different systems of stress factors? In: Global \& Regional Ecological Problems, R.G. Khlebopros (Ed.), Krasnoyarsk State Technical University Publ., 1994, 499-516.

[29] A.S. Mansurov, T.P. Mansurova, E.V. Smirnova, L.S. Mikitin, A.V. Pershin. Human adaptation under influence of synergic system of factors (treatment of oncological patients after operation). Institute of Biophysics Russian Academy of Sciences, Preprint 212B Krasnoyarsk, 1995.

[30] R.N. Mantegna. Hierarchical structure in financial markets. The European Physical Journal B, 11, No. 1 (1999), 193-197.

[31] S.M. Markose. Computability and evolutionary complexity: markets as complex adaptive systems (CAS). Economic Journal, 115 (2005), F159-F192. Available online at SSRN: $\mathrm{http}: / /$ ssrn.com/abstract $=745578$

[32] R.N. Mantegna, H.E. Stanley. An introduction to econophysics: correlations and complexity in finance. Cambridge University Press, Cambridge, 1999. 
[33] D. Matesanz, G.J. Ortega. Network analysis of exchange data: Interdependence drives crisis contagion, MPRA Paper No. 7720, posted 12 March 2008, Online at http://mpra.ub.unimuenchen.de/7720/

[34] R. McCarty, K. Pasak. Alarm phase and general adaptation syndrome. In: Encyclopedia of Stress, George Fink (ed.), Vol. 1, Academic Press, 2000, 126-130.

[35] I. Meric, G. Meric. Co-movements of European equity markets before and after the 1987 crash. Multinational Finance J., 1, No. 2 (1997), 137-152.

[36] E.P. Odum. Fundamentals of ecology (3d ed.). W. B. Saunders, Comp., Philadelphia - London - Toronto, 1971.

[37] J. Oechssler, F. Riedel. On the dynamic foundation of evolutionary stability in continuous models. J. of Economic Theory, 107 (2002), 223-252.

[38] J.-P. Onnela, A. Chakraborti, K. Kaski, J. Kertész, A. Kanto. Dynamics of market correlations: Taxonomy and portfolio analysis. Physical Review E, 68 (2003), 056110.

[39] M.Y. Ozden. Law of the minimum in learning. Educational Technology \& Society, 7 , No. 3 (2004), 5-8.

[40] Q. Paris. The return of von Liebig's “Law of the minimum”. Agron. J., 84 (1992), 1040-1046

[41] K. Pearson. On lines and planes of closest fit to systems of points in space. Philosophical Magazine, 2, No. 6 (1901), 559-572.

[42] V. Plerou, P. Gopikrishnan, B. Rosenow, L.A.N. Amaral, T. Guhr, H.E. Stanley. Random matrix approach to cross correlations in financial data. Phys. Rev. E, 65 (2002), 066126.

[43] L.I. Pokidysheva, R.A. Belousova, E.V. Smirnova. Method of adaptometry in the evaluation of gastric secretory function in children under conditions of the North. Vestn. Ross Akad Med Nauk, No. 5 (1996), 42-45. PMID: 8924826

[44] L.D. Ponomarenko, E.V. Smirnova. Dynamical characteristics of blood system in mice with phenilhydrazin anemiya. Proceeding of 9th International Symposium "Reconstruction of homeostasis", Krasnoyarsk, Russia, March 15-20, 1998, vol. 1, 42-45.

[45] M. Potters, J.P. Bouchaud, L. Laloux. Financial applications of random matrix theory: old laces and new pieces. Acta Phys. Pol. B, 36, No. 9 (2005), 2767-2784.

[46] V.N. Razzhevaikin, M.I. Shpitonkov. Substantiation of correlation adaptometry based on evolutionary optimality principles. Computational Mathematics and Mathematical Physics, 43, No. 2 (2003), 296-307.

[47] R.T. Rockafellar. Convex analysis. Princeton University Press, Princeton, NJ, 1970. Reprint: 1997. 
[48] F. Salisbury. Plant physiology (4th ed.). Wadsworth Belmont, CA, 1992.

[49] J.K. Schkade, S. Schultz. Occupational adaptation in perspectives. Ch. 7 in: Perspectives in Human Occupation: Participation in Life, By Paula Kramer, Jim Hinojosa, Charlotte Brasic Royeen (eds), Lippincott Williams \& Wilkins, Baltimore, MD, 2003, 181-221.

[50] K.R. Sedov, A.N. Gorban', E.V. Petushkova (Smirnova), V.T. Manchuk, E.N. Shalamova. Correlation adaptometry as a method of screening of the population. Vestn. Akad Med Nauk SSSR, No. 10 (1988), 69-75. PMID: 3223045

[51] H. Selye. Adaptation energy. Nature, 141 (3577) (21 May 1938), 926.

[52] H. Selye. Experimental evidence supporting the conception of "adaptation energy". Am. J. Physiol., 123 (1938), 758-765.

[53] A.M. Sengupta, P.P. Mitra. Distributions of singular values for some random matrices. Phys. Rev. E, 60 (1999), 3389-3392.

[54] F.N. Semevsky, S.M. Semenov. Mathematical modeling of ecological processes. Gidrometeoizdat, Leningrad, 1982.

[55] P.G. Shumeiko, V.I. Osipov, G.B. Kofman. Early detection of industrial emission impact on Scots Pine needles by composition of phenolic compounds. In: Global \& Regional Ecological Problems, R.G. Khlebopros (Ed.), Krasnoyarsk State Technical University Publ., 1994, 536543.

[56] R. Smith. The spread of the credit crisis: view from a stock correlation network (February 23, 2009). Available online at SSRN: http://ssrn.com/abstract=1325803

[57] S.O. Strygina, S.N. Dement'ev, V.M. Uskov, G.I. Chernyshova. Dynamics of the system of correlations between physiological parameters in patients after myocardial infarction. In: Mathematics, Computer, Education, Proceedings of Conference, Issue 7, Moscow, 2000, 685-689.

[58] G.N. Svetlichnaia, E.V. Smirnova, L.I. Pokidysheva. Correlational adaptometry as a method for evaluating cardiovascular and respiratory interaction. Fiziol. Cheloveka, 23, No. 3 (1997), 58-62. PMID: 9264951

[59] A.V. Vasil'ev, G.Iu. Mal'tsev, Iu.V. Khrushcheva, V.N. Razzhevaikin, M.I. Shpitonkov. Applying method of correlation adaptometry for evaluating of treatment efficiency of obese patients. Vopr. Pitan., 76, No. 2 (2007), 36-38. PMID: 17561653

[60] M.J. West-Eberhard. Developmental plasticity and evolution. Oxford University Press, US, 2003.

[61] E. Zuckerkandl, R. Villet. Concentration-affinity equivalence in gene regulation: convergence of genetic and environmental effects. PNAS USA, 85 (1988), 4784-4788. 